Elsevier Editorial System(tm) for Journal of Neuroimmunology

Manuscript Draft

Manuscript Number: JNI-D-13-00056R2

Title: Detection of auto-antibodies to DAT in the serum: interactions with DAT genotype and psychostimulant therapy for ADHD.

Article Type: Original Article

Section/Category: Clinical Neuroimmunology

Keywords: Auto-antibodies (aAbs) to neuro-receptors; Auto-immunity in neuro-psychiatry; Conners' scale; CGAS; Dopamine Transporter (DAT); methylphenydate; 10-repeat allele; 9-repeat allele; ELISA.

Corresponding Author: Dr. Walter Adriani,

Corresponding Author's Institution: Istituto Superiore di Sanita'

First Author: Grazia Giana

Order of Authors: Grazia Giana; Emilia Romano; Maria Cristina Porfirio; Roberto D'Ambrosio; Silvia Giovinazzo; Miriam Troianiello; Eleonora Barlocci; Domenica Travaglini; Oleg Granstrem; Esterina Pascale; Luigi Tarani; Paolo Curatolo ; Giovanni Laviola; Walter Adriani

Abstract: Interest is rising for auto-immune contribution in neuro-psychiatry. We evaluated the autoantibodies against dopamine transporter (DAT aAbs) in 63 children (48 who met DSM-IV-TR criteria, 15 healthy controls). Methods. ADHD patients were assigned, according to severity as recruited, either to a non-pharmacological therapy (NPT) or to a pharmacological treatment (PT) with methyilphenidate (MPH). In ADHD children, blood samples were withdrawn at recruitment (T0 basal) and after a 6-week period (T1), and we also characterized DAT genotype (9-repeat or 10-repeat alleles; final $n=30$ ). After 18 months of NPT or PT, some patients (carrying at least one 9-repeat allele) were blood sampled again (T2), for comparison with healthy controls (final $\mathrm{n}=8$ ). Results. Compared to NPT, basal DAT aAbs titers were higher within most severe patients (then assigned to PT), specifically if carrying a DAT 10/10 genotype. DAT aAbs levels of NPT group resulted highly correlated with distinct subscales of Conners' Parent / Teachers Scales (Rs > 0,34), especially within DAT 10/10 genotype (Rs $>0,53$ ). While T1 titers were elevated over T0 baseline for NPT children, such an increase was not observed in PT patients carrying at least one 9-repeat allele, who also showed behavioral response to subchronic MPH. After 18-months MPH exposure, DAT aAbs titers in PT subjects were comparable to those of healthy controls, while titers remained significantly elevated in NPT patients. Data warrant further research on serum DAT aAbs, which could be used to confirm ADHD diagnosis and/or to monitor therapeutic efficacy of MPH. 

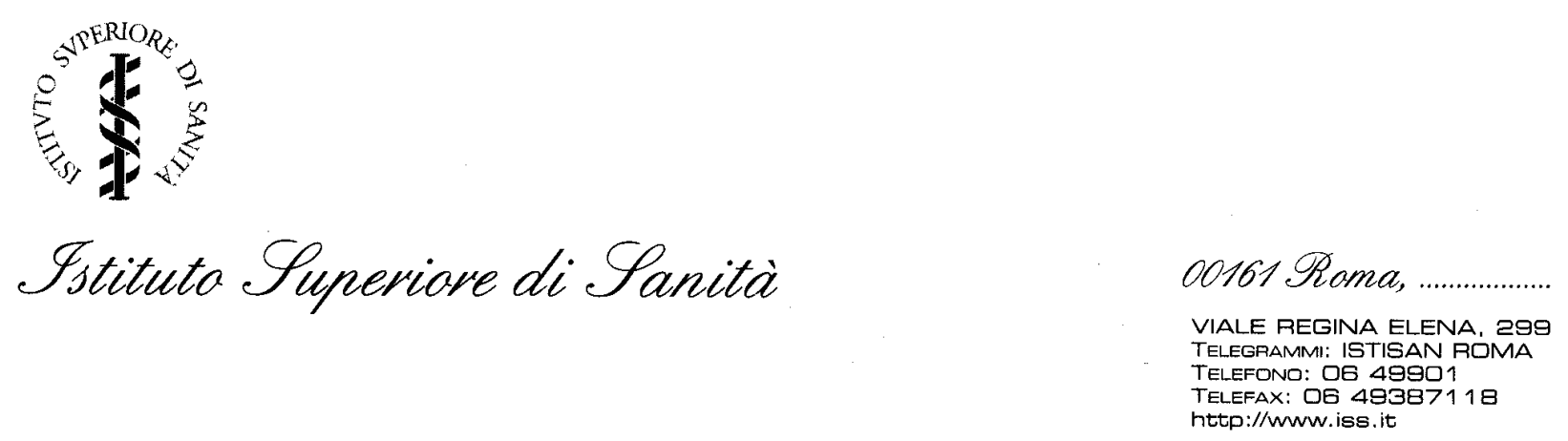

\section{Cover Letter}

Dear editor,

Please find enclosed a paper where we show that the immune system may provide intriguing new bio-markers for neuro-psychiatric symptoms during development. Specifically, we found that serum titers of a circulating auto-antibody directed to the dopamine transporter (DAT) might be related to the modulation of ADHD symptoms, like inattention, impulsive and oppositional-defiant behavior. This finding opens new avenues for the evidence-based diagnostics (and, possibly, for monitoring of a psychostimulant-based therapy) in children with the ADHD syndrome.

We explicitly state that neither the manuscript nor any of its parts have been published before, and are also not being considered for publication elsewhere. We are pleased to suggest the following five referees:

Prof. Pietro PANEI, pietro.panei@iss.it Istituto Superiore di Sanità, Rome, IT.

Dr. Roberta Magliozzi Roberta.Magliozzi@iss.it Istituto Superiore di Sanità, Rome, IT.

Dr. Davide MARTINO, davidemartino@virgilio.it Neurological \& Psychiatric Sci., Univ. Bari, IT.

Prof. Luigi TARANI, luigi.tarani@uniroma1.it Pediatry, "La Sapienza” University, Rome, IT.

Dr. Aimé RAVEL, aime.ravel@institutlejeune.org Foundation « Jerome Lejeune », Paris, FR.

Dr Walter ADRIANI

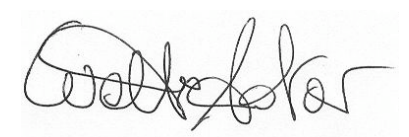

Istituto Superiore di Sanita' - Roma (Italia)

Tel: +39064990 3171 - Fax: +39064957821 
Reviewer \#1: I appreciate that the authors now have expanded the number of healthy controls. I still think on a number of points the paper needs to be revised.

1. The paper now deals with a variety of objectives which are not well connected. For example the section relating DAT genotype to response to $\mathrm{MPH}$ in my view should not be part of the present paper. Please focus on the role of aAb in relation to $A D H D$ severity as well as in comparison to healthy controls. You can try to break down the aAb results by genotype, but PLEASE include the precise results. The current vague wording is not acceptable with regard to results.

R. We thank the referee for the additional comments. We only partially agree with the suggestion to remove the section relating DAT genotype to MPH response. We think it is important to underline the role of DAT genotype in this paper for two reasons.

-First, we confirm what already reported in literature i.e. association of the DAT1 and ADHD symptoms (Cook et al. 1995, replicated by Gill et al. 1997). The DAT1 high-risk allele (i.e. the 10-repeat allele) can explain $1 \%-4 \%$ of the overall variance in ADHD symptoms; its relation with hyperactive-impulsive symptoms is stronger and more reliable than that with inattentive symptoms (Waldmann et al. 1998). In children carrying both DAT1 high-risk alleles, therefore, the severity of the disorder may be influenced.

-Further, the DAT1 high-risk alleles may partially explain why as many as $30 \%$ of ADHD children do not respond to psychostimulant medications (Madras et al. 2002).

However, to meet referee's comment, we have rephrased this issue in both Introduction and Discussion, and focused a bit more on the correlations between aAbs and behavioral symptoms.

2. The results section is much too vague. Instead of writing "As a whole we report abnormal levels of Aab in severe ADHD patients" we need to see the precise actual numbers and defitinions.

R. As requested by the reviewer, we have rephrased this sentence, and report that highest levels of aAbs are seen in more severe ADHD patients. We actually present, in this paper, five figures and three correlation's tables. Indeed, we made all effort to provide a precise representation of data in the text, as far as possible.

3. It is completely unclear why 16 subjects needed to be removed.

R. We have now provided a clear explanation of why 16 subjects were removed. Indeed, 13 of these subjects presented: "altered values detected in standard blood screen at T0". In particular, they have been excluded because of an increase in the percentage of lymphocytes and/or eosinophils in total number of white blood cells. Increased levels of lymphocytes are frequently caused by recent infections or infestations of various nature (viral, bacterial, parasites, allergies, etc.); they represent the aspecific expression of activation of the immune system. Since we are measuring an autoimmune (aAbs) parameter, exclusion of patients with immune activation was indeed one of the explicit, declared conditions for patient's enrollment in this study (see Methods).

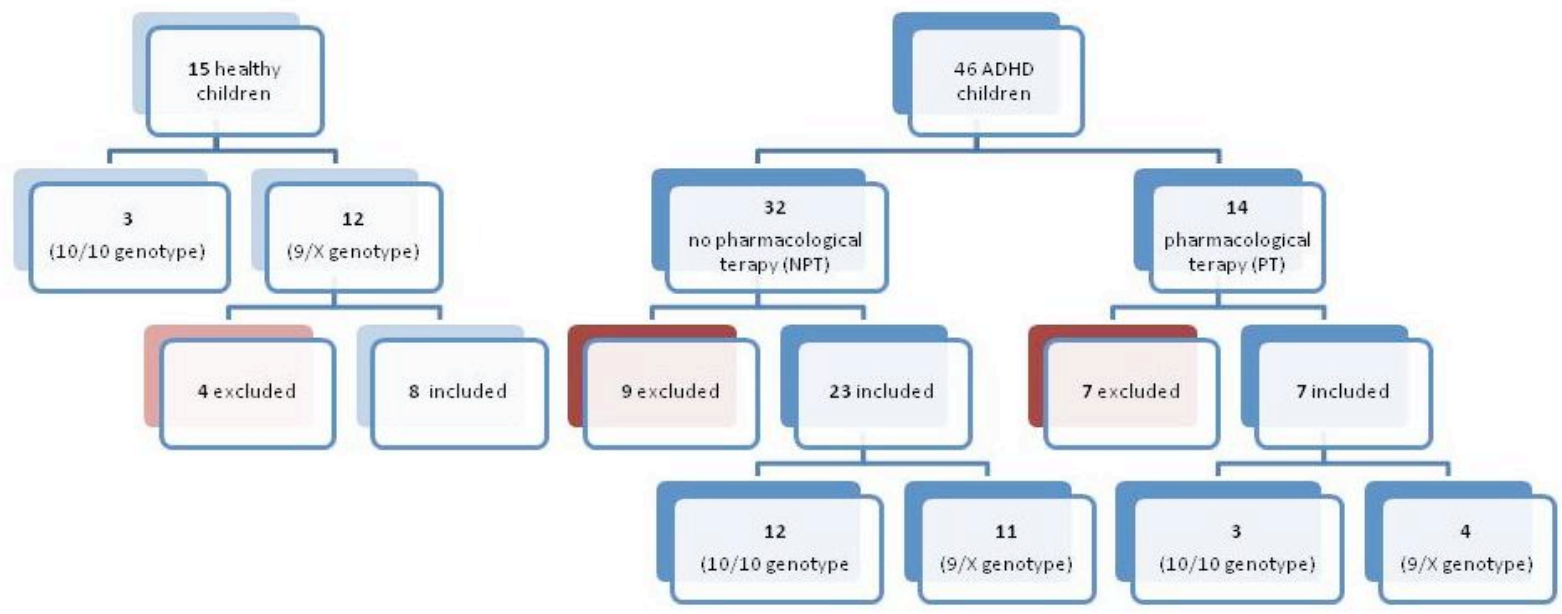

Three other subjects were excluded: two because they were switched to atomoxetine treatment instead of MPH, one because (s)he was not belonging to Caucasians. The diagram shows the number of children we enrolled. 
I would recommend the authors to reconsider the structure of the paper and focus more on the cross-sectional findings, which should be described precisely. Why after all would treatment with MPH lead to Aab alterations?

R. This is of course a very pertinent question; but data available do not allow us to provide a clear answer yet. We can try to speculate on what might be the mechanism.

It is known that abnormal dopamine levels and deregulation of dopaminergic components expressed in immune cells are associated with some autoimmune disorders (Pacheco et al. 2014). Dopamine has been hypothesized to activate T-cell function indirectly, by suppressing T-regulatory cell (Treg) (Cosentino et al. 2007; Kipnis et al. 2004, Levite 2008). Treg is involved in autoimmunity, and secretes IL-10 and TGF-b. Due to its inhibitory properties, an altered activity of Treg could impair $\mathrm{T}$ cell immunity and promote development or persistence of infectious agents in the organism. In contrast, a reduced activity of Treg (due to abnormal dopamine) could lead to uncontrolled activation and function of effector $T$ cells and to the onset of autoimmunity (Pacheco et al. 2010). Furthermore, at certain concentrations, dendritic-cell derived dopamine promotes IL-23 production and, thereby, enhances Th17 responses with inflammation (Pacheco et al. 2014).

Based on these evidences, it is thought that dopamine can mediate communication between immune cells, and the cross-talk between the immune and the nervous system (Pacheco et al. 2014, Buttarelli et al. 2011, Nakano et al. 2009, Cosentino et al. 2007, Sarkar et al. 2010). Thus, over-expression of DAT in lymphocytes of ADHD children could imply a dysregulation of immune and neuro-immune systems. The blockade of DAT, by MPH, may increase and normalize the dopamine levels, which act on the lymphocytes. It was reported, consistently, that the MPH may induce (i) a hyperactivity of the immune system and a heightened tendency to respond to antigenic or immunogenic stimuli, (ii) hypergammaglobilinemia and (iii) reduction of T-helper/inducer cells (Auci et al. 1997).

We hypothesize that the MPH may be competing with auto-antibodies for binding to the DAT sequence. In this manner, the drug may well regulate the increase of DAT aAbs.

We are reporting below the list of references added to the text:

- Auci DL, Fikrig S, Rodriquez J (1997) Methylphenidate and the immune system. J Am Acad Child Adolesc Psychiatry 36(8):1015-6

- Buttarelli FR, Fanciulli A, Pellicano C, Pontieri FE (2011) The dopaminergic system in peripheral blood lymphocytes: from physiology to pharmacology and potential applications to neuropsychiatric disorders. Curr Neuropharmacol. 9(2):278-88

- Cook EH Jr, Stein MA, Krasowski MD, Cox NJ, Olkon DM, Kieffer JE, Leventhal BL (1995) Association of attentiondeficit disorder and the dopamine transporter gene. Am J Hum Genet 56:993-998

- Cosentino M, Fietta AM, Ferrari M, Rasini E, Bombelli R, Carcano E, Saporiti F, Meloni F, Marino F, Lecchini S (2007) Human CD4+CD25+ regulatory $T$ cells selectively express tyrosine hydroxylase and contain endogenous catecholamines subserving an autocrine/paracrine inhibitory functional loop. Blood. 15;109(2):632-42

- Gill M, Daly G, Heron S, Hawi Z, Fitzgerald M (1997) Confirmation of association between attention deficit hyperactivity disorder and a dopamine transporter polymorphism. Mol Psychiatry 2:311-313

- Kipnis J, Cardon M, Avidan H, Lewitus GM, Mordechay S, Rolls A, Shani Y, Schwartz M (2004) Dopamine, through the extracellular signal-regulated kinase pathway, downregulates CD4+CD25+ regulatory T-cell activity: implications for neurodegeneration. J Neurosci. 7;24(27):6133-43

- Levite M (2008) Neurotransmitters activate T-cells and elicit crucial functions via neurotransmitter receptors. Curr Opin Pharmacol. 8(4):460-71

- Madras BK, Miller GM, Fischman AJ (2002) The dopamine transporter: relevance to attention deficit hyperactivity disorder (ADHD). Behav Brain Res. 2002 Mar 10;130(1-2):57-63

- Nakano K, Higashi T, Takagi R, Hashimoto K, Tanaka Y, Matsushita S (2009) Dopamine released by dendritic cells polarizes Th2 differentiation. Int Immunol. 21(6):645-54.

- Pacheco R, Riquelme E, Kalergis AM (2010) Emerging evidence for the role of neurotransmitters in the modulation of $T$ cell responses to cognate ligands. Cent Nerv Syst Agents Med Chem. 10(1):65-83.

- Pacheco R, Contreras F, Zouali M (2014) The dopaminergic system in autoimmune diseases. Front Immunol. 21;5:117

- Sarkar C, Basu B, Chakroborty D, Dasgupta PS, Basu S (2010) The immunoregulatory role of dopamine: an update. Brain Behav Immun 24(4):525-8

- Waldman ID, Rowe DC, Abramowitz A, Kozel ST, Mohr JH, Sherman SL, Cleveland HH, Sanders ML, Gard JM, Stever C (1998) Association and linkage of the dopamine transporter gene and attention-deficit hyperactivity disorder in children: heterogeneity owing to diagnostic subtype and severity. Am J Hum Genet 63(6):1767-76 


\section{HIGHLIGHTS}

In $30 \mathrm{ADHD}$ and 8 healthy children, serum auto-antibodies directed against DAT were found. DAT aAbs were elevated in serum of most severe ADHD patients, needing MPH medication. In patients with DAT 9-repeat allele, MPH was active both on symptoms and DAT aAbs titer. In DAT 10/10 patients, anti-DAT titers correlated with all subscales of Conners' (Rs $>0,34)$. Serum DAT aAbs could be used to confirm ADHD diagnosis / to monitor treatment efficacy. 


\title{
Detection of auto-antibodies to DAT in the serum: interactions with DAT genotype and psycho-stimulant therapy for ADHD.
}

\author{
Grazia Giana * (1, 3), Emilia Romano * (2, 3), Maria Cristina Porfirio (1), Roberto \\ D’Ambrosio (4), Silvia Giovinazzo (1), Miriam Troianiello (1), Eleonora Barlocci (2), \\ Domenica Travaglini (2), Oleg Granstrem (5), Esterina Pascale (6), Luigi Tarani (4), Paolo \\ Curatolo (1), Giovanni Laviola (2), and Walter Adriani (2).
}

- (1) Child Neuro-Psychiatry Unit - University of "Roma 2" Tor Vergata, Rome, Italy

- (2) Cell Biology \& Neurosciences Dept. - Istituto Superiore di Sanità, Rome, Italy

- (3) Bambino Gesù Children's Hospital IRCCS, Rome, Italy

- (4) Policlinico Universitario "Umberto I" - "Sapienza" University of Rome, Rome, Italy

- (5) National BioService LLC, Saint-Petersburg, Russia

- (6) Medical Surgical Sciences \& Biotechnology Dept. - "Sapienza” University of Rome, Rome, Italy

$\left({ }^{*}\right)$ Both authors contributed equally to this work.

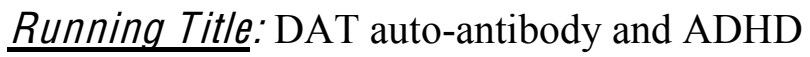

Corresponding Author: Walter Adriani, $\mathrm{PhD}$ (at above address)

Tel. +390649903171 -- Fax. +3906495 7821 -- e-mail: adriani@iss.it

Acknowledgements: Research received support from EU project "EMTICS" (Seventh Framework Programme FP7 / 2007-2013), agreement n 278367 (partner grant to G.L.); EU project "NeuroGenMRI” (ERA-net "PrioMedChild”), Italian Ministry of Health (partner grant to W.A.); “Gambling”, Department of Antidrug Policies, Council of Ministries' Presidency, Italy (grant to G.L. and W.A.). We warmly acknowledge the precious help of Dr Rosanna Mancinelli for her continued theoretical and practical support. There is no conflict of interest to disclose. 


\section{SUMMARY}

Interest is rising for auto-immune contribution in neuro-psychiatry. We evaluated the autoantibodies against dopamine transporter (DAT aAbs) in 61 children (46 ADHD who met DSM-IVTR criteria, 15 healthy controls). Methods. ADHD patients were assigned, according to severity, either to a non-pharmacological therapy (NPT, N=32) or to a pharmacological treatment (PT, $\mathrm{N}=14$ ) with methyilphenidate (MPH). In ADHD children, blood samples were withdrawn twice, at recruitment (T0 basal) and after 6 weeks (T1); following 16 excluded subjects, DAT genotype was characterized (9-repeat or 10-repeat alleles; N=15 each). After 18 months of NPT or PT, some patients (carrying at least one 9-repeat allele) were blood sampled again (T2), for comparison with healthy controls (final $\mathrm{n}=8$ ). Results. Compared to NPT, basal DAT aAbs titers were higher within most severe patients (then assigned to PT), specifically if carrying a DAT 10/10 genotype. DAT aAbs levels of NPT group resulted highly correlated with distinct subscales of Conners' Parent / Teacher Scales $(\mathrm{Rs}>0.34)$, especially within DAT 10/10 genotype (Rs $>0.53)$. While T1 titers were elevated over T0 baseline for NPT children, such an increase was not observed in PT patients carrying at least one 9-repeat allele, who also showed behavioral response to subchronic MPH. After 18-months MPH exposure, DAT aAbs titers in PT subjects were comparable to those of healthy controls, while titers remained significantly elevated in NPT patients. Data warrant further research on serum DAT aAbs, which could be used to confirm ADHD diagnosis and/or to monitor therapeutic efficacy of MPH.

Key Words: Auto-antibodies (aAbs) to neuro-receptors; Auto-immunity in neuro-psychiatry; Conners' scale; CGAS; Dopamine Transporter (DAT); methylphenydate; 10-repeat allele; 9-repeat allele; ELISA. 


\section{INTRODUCTION}

Attention Deficit/Hyperactivity Disorder (ADHD) has been internationally recognized as a medical neuro-developmental condition (Curatolo et al., 2010; Davis et al., 2011; Purper-Ouakil et al., 2011; Cortese, 2012); global interest in long-term consequences of ADHD and of psychostimulant administration for ADHD is on the rise (Hinshaw et al., 2011). According to the current criteria of Diagnostic and Statistical Manual of Mental Disorders, $4^{\text {th }}$ edition- Text Revision (DSM IV-TR), ADHD prevalent symptoms include problems in maintaining attention, excessive motor activity, and impulsivity, which often lead to poor academic performance and impaired social interactions (American Psychiatric Association, 2000). These symptoms develop early in up to 5\% of children (Polanczyk et al., 2007), and can persist into adolescence and adulthood (Biederman et al., 2006). Frequently comorbid with ADHD are other impulse-control disorders, like oppositional defiant disorder, conduct disorder, substance abuse and/or dependence problems (Hollander et al., 2000, 2005), pathological gambling, all of which may be conceptualized as part of the addictive disorder spectrum (Fontenelle et al., 2011).

Although the multi-factorial aetiology of ADHD is still unclear, evidence suggests that the disorder is linked to imbalanced levels of dopamine neurotransmitter. Some accounts present ADHD as a motivational dysfunction (Sonuga-Barke, 2005), arising from altered processes within fronto-striatal circuits (Sagvolden \& Sergeant, 1998; Oades, 1998; Chamber \& Potenza, 2003). For this reason, one focus of ADHD research has converged on the brain dopamine transporter (DAT) in the clinics and in preclinical models. It has been proposed that specific ADHD symptoms may arise from a modification in DAT expression and function (Jucaite et al., 2005; Bannon, 2005; Berridge et al., 2005): human DAT1 gene has a VNTR polymorphism of $40 \mathrm{bp}$ (3-11 repeats) in the 3' untranslated region, with 9- and 10-repeat variants being the most common in Caucasians. 10-repeat VNTR polymorphism of DAT has been associated with ADHD (Cook et al., 1995; Gill et al., 1997), with obsessive-compulsive disorder and with Tourette's syndrome (McElroy \& Kelsoe, 2013; Sharp etal. 2009). DAT1 high-risk allele (i.e. the 10-repeat allele) can explain 1\%$4 \%$ of the overall variance in ADHD symptoms; its relation with hyperactive-impulsive symptoms is stronger and more reliable than that with inattentive symptoms (Waldmann et al. 1998). 
However, although the presence of DAT1 high-risk alleles may influence the severity of the disorder and may explain why as many as 30\% of ADHD children do not respond to psychostimulants (Madras et al. 2002), other non-genetic factors are crucial for the onset of ADHD symptoms. To account for a possible DAT alteration, a rising interest exists for auto-immune processes and psycho-immunological interactions (Shulman, 2009; Graus et al., 2010). A breach in blood brain barrier (BBB) integrity, due to conditions of stress (Kuang et al., 2004) or owing to a traumatic injury to the brain (like e.g. as complication of a difficult delivery, Ankeny \& Popovich, 2010), could implicate the draining of CNS antigens to peripheral lymphoid organs, with subsequent auto-immune responses (Diamond et al., 2009; Levin et al., 2010). According to recent literature, anti-neuronal antibodies may target a wide range of CNS proteins, including neuroreceptors (Davies et al., 2007; Graus et al., 2008; Zuliani et al., 2012). Behavioral dysfunction might possibly stem from anti-neuronal auto-antibodies (aAbs) that would presumably compromise neural function (Granstrem et al., 2006). Circulating aAbs against neuro-receptors are reliable biomarkers for Systemic Lupus Erythematosus (Solal \& Diamond, 2011), intractable seizures (Rogers et al., 1994; Twyman et al., 1995), brain ischemic stroke (Dambinova et al., 2003), Hashimoto's encephalopathy (Chong et al., 2003) and Sydenham's chorea. Other two neurological conditions with a claimed role for aAbs and related neuro-psychiatric symptoms are the Limbic and the NMDAR-Ab encephalitis. While seizures are prominent with GABAB receptor (GABAB-R) aAbs, there may be psychiatric features with AMPA receptor (AMPA-R) aAbs (Lai et al., 2009; Lancaster et al., 2010; Zandi et al. 2010; Graus et al., 2010) and with NMDA receptor (NMDA-R) aAbs (Dalmau et al., 2011). Many of these patients are children who may initially seek for psychiatric wards for acute anxiety, behavioral change or psychosis. Interestingly, a role for autoimmunity in general and for aAbs in particular has been claimed for Tourette's (Hoekstra \& Minderaa, 2005; Martino et al., 2009; Rizzo et al., 2010), for obsessive-compulsive disorder (Teixeira et al., 2014), and for ADHD as well (Passarelli et al., 2013; Hegvik et al., 2014).

Circulating aAbs to CNS antigens can be also detected in animal models (Dambinova et al., 1997, 1998; Vincent et al., 1999; Kowal et al., 2006; Knight łt al., 2007; Colasanti et al., 2009; Capone et al., 2008), as well as in opiate-treated mice (Granstrem et al., 2006). In this line, we recently proposed (Adriani et al., 2012) that a DAT altered turnover / degradation ratio may lead to 
an over-production of neuro-receptor fragments, which might then overcome the BBB and spill into the blood, where they can generate an auto-immune reaction. This auto-immune challenge could in turn lead to an enduring and possibly detectable interference with the dopamine (DA) neuro-transmission as well as to DA-related behavioral changes, like ADHD symptoms (i.e. impulsivity and hyperactivity). Thus, purpose of the present study was 1) to ascertain the presence in the blood of circulating auto-antibodies (aAbs) targeting some epitopes of the DAT protein, 2) to measure levels of such DAT aAbs, by means of an ELISA assay, as a function of therapy with or without pharmacological MPH treatment, 3) to correlate these DAT aAbs titers with clinical scores of ADHD symptoms, and 4) to further evaluate the above parameters as a function of the individual DAT genotype. We hypothesized that a more clear account of observed symptoms could be served by taking into consideration the interaction between genetic and autoimmune parameters.

\section{MATERIAL AND METHODS}

\subsection{Recruitment of Patients and Healthy Controls}

Participants included 61 children. We recruited 48 patients with a formal diagnosis of ADHD, with a female to male ratio of 1:5, referred to Child Psychiatry Unit of Tor Vergata University from April 2010 to March 2012. Two of these recruited ADHD children were dropped out, since they turned out to be non-responders to the drug; we also recruited 15 healthy children (handled under the same routine conditions from April 2013 to March 2014, in the same period as the patients' resampling, see below) to act as controls. All subjects had a full Scale IQ over 84, as assessed by the Wechsler Intelligence Scale - III edition (Wechsler, 1991). They were evaluated by child neuropsychiatrists who determined the diagnosis of ADHD, according to DSM IV-TR criteria (American Psychiatric Association, 2000); a medical work-up excluded any other neuro-genetic disease or immune disorder, as well as any psychiatric comorbidity (conduct disorder, obsessive-compulsive disorder, Tourette, depression, bipolar disorder, psychosis), assessed by the Schedule for Affective Disorders and Schizophrenia for School Age Children - Present and Lifetime Version (K-SADS- 
PL) (Kaufman, 1997). Post-recruitment exclusions were also made for coeliac or diabetic disease, and in case of recent fever or allergy, as resulting from standard blood screen.

The clinical sample was divided into two, based on therapeutic intervention decided at enrollment: children with milder symptoms, which did not need pharmacological treatment, underwent cognitive-behavioral therapy and/or periodic follow-up (unmedicated, NPT group; $\mathrm{N}=32$ ); severe children, with a significant impairment of adaptive functioning in different areas of life, were assigned to pharmacological treatment with MPH (PT group; N=14).

The study was formally approved by ISS Ethical Committee (Prot. CE-ISS 09/270 of 15 July 2009, scientific responsible and PI: W.A.). Informed consent procedures included searching for consent from the child (using age-adequate approaches) and illustrating to parents the standard consent form; the parents gave their written informed consent for the child to participate in this study. All potential participants who decided not to participate in the study were not disadvantaged in any way by not participating. Also, we declare that collected biological materials were used solely to the purpose of this study; the responsible person for paediatric privacy is one of authors (M.C.P.). The rules set by the Code of Ethics of the World Medical Association (Declaration of Helsinki), which has been printed in the British Medical Journal (18 July 1964), were respected.

\subsection{Clinical Assessment}

Each patient was evaluated by trained child neuro-pyschiatrists at our Unit, according to the DSM-IV and ICD-10 criteria for ADHD. Information was gathered from the clinical interviews and questionnaires with the parents, teacher and from direct observations of the patients.

Parents completed SNAP-IV that elicits DSM-IV TR criteria for ADHD on a four-point scale of frequency (Swanson et al., 1983), also giving information about ADHD subtypes (inattentive, hyperactive-impulsive, combined type). ADHD symptoms were also determined using Conners' Parent Rating Scale; each item was scored according to the published measure from 0 (Not true at all) to 3 (Very much true) (Conners, 1998). The semi-structured Schedule for Affective Disorders and Schizophrenia - Present and Lifetime version (K-SADS / PL) and also the Child Behavior Checklist/4-18 (CBCL; Achenbach, 1991) were used separately, to elicit parents' and patients' reports of signs and symptoms that might indicate possible co-morbidities. 
The Children's Global Assessment Scale (CGAS) was used by clinicians to measure the overall severity of social and psychiatric functioning for children ages 4-16 years (Shaffer et al., 1983) CGAS scores range between 1 and 100, with higher scores indicating better functioning. The full clinical assessment was performed at time of recruitment, while both parents completed the Conners' Parent Rating Scale and clinicians completed the CGAS at time T0 and T1. The patient's teacher completed (only once, at time of recruitment) the Conners' Teacher Rating Scale.

\subsection{Biochemical and Genetic Assessment}

After recruitment, in the 46 ADHD children we were able to collect two blood samplings. Time TO: basal withdrawal, at recruitment (early in the morning and/or without breakfast, i.e. when stomach was empty; also for standard blood screen); Time T1: second withdrawal, taken either after 6 weeks from time T0 (for subjects who did not undergo pharmacological therapy, NPT group) or after 6 weeks from when MPH had reached the therapeutic dose (for subjects which were assigned, at recruitment, under pharmacological treatment, PT group). Blood was withdrawn from patients using standard phlebotomy procedures: it was collected in a sterile 10-ml plain centrifuge tube and allowed to clot for 5-10 minutes, then centrifuged for $10 \mathrm{~min}$ at $1500 \mathrm{rpm}$ in a standard centrifuge. The serum was stored at $-80^{\circ} \mathrm{C}$ until use.

Of 46 patients, 16 children were excluded a posteriori, due to recent fever / allergy before blood sampling, as altered values were detected in standard blood screen at T0. Ten children had higher lymphocytes count ( $41.0 \pm 0.84 \%$, up to $47.0 \%$ ) and seven children had higher eosinophils

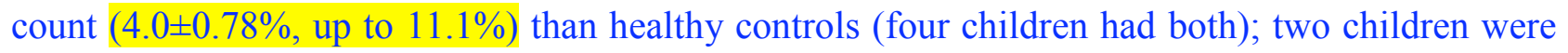
classified as non responders to MPH and switched to atomoxetine treatment; one child was also excluded since he was the only one not belonging to Caucasian ethnicity. In the remaining children $(\mathrm{N}=30)$, we collected buccal samples using a Catch-all sample collection Swab (Epicentre), in order to verify specifically the DAT 1 gene VNTR polymorphism in the 3' untranslated region (3'UTR), rs 28363170 (Sano et al., 1993; Vandenbergh et al., 1992). Of these, 15 had a DAT 10/10 genotype while 15 were carrying at least one 9-repeat allele.

We recruited 15 subjects as healthy controls. Of these, only 3 had a DAT 10/10 genotype (too few to be further assessed) while 12 were carrying at least one 9-repeat allele. After excluding 4 
children with recent fever or allergy, we ended up with 8 healthy subjects. Hence, ADHD children with at least one 9-repeat allele were further contacted for re-sampling, after one-to-two years (average: 18 months) of exposure to MPH or to unmedicated conditions (PT or NPT, respectively). For the latter, we were able to involve just 4 patients (out of 11). We aimed to re-assess their DAT aAbs titers (time T2), to be compared with healthy controls; therefore, in addition to T0 baseline, blood samples were taken twice in these children, months apart, to improve reliability of findings. All these subjects were withdrawn blood samples in the same conditions as for time T0.

\subsection{PCR methods}

Genomic DNA was prepared from buccal swab samples by using the BuccalAmp ${ }^{\mathrm{TM}}$ DNA Extraction Kit, following the manufacturer instructions (Epicentre, USA). Briefly, after collecting buccal cells, the swab end was placed into a tube containing QuickExtract DNA extraction solution and rotated a minimum of five times. The tube was vortex mixed for 10 seconds and incubated at $65^{\circ} \mathrm{C}$ for 1 minute. After vortex mix for 15 seconds, the tube was transferred to $98^{\circ} \mathrm{C}$ and incubated for 2 minutes. After vortex mix for 15 seconds, the DNA was stored at $-20^{\circ} \mathrm{C}$ until processing. The yield of DNA is usually between 2-14 $\mathrm{ng} / \mu \mathrm{l}$.

The 3'-UTR repeated sequence of the DAT1 gene was amplified by the polymerase chain reaction (PCR). The primer sequences employed were 5'-TGT GGT GTA GGG AAC GGC CTG AG-3' (DAT1-F) and 5'-CTT CCT GGA GGT CAC GGC TCA AGG-3' (DAT1-R). The PCR amplification was carried out in a final volume of $50 \mu 1$ containing $3 \mu 1$ of genomic DNA prepared using the Buccal Amp DNA extraction kit, $1.5 \mathrm{mM} \mathrm{MgCl} 2,200 \mu \mathrm{M}$ dNTP, $50 \mathrm{mM} \mathrm{KCl}, 10 \mathrm{mM}$ Tris$\mathrm{HCl}$ (pH8.3), $0.25 \mu \mathrm{M}$ of each primer, and $1 \mathrm{U}$ of Promega Taq DNA polymerase. The PCR amplification was performed for 35 cycles consisting of $94^{\circ} \mathrm{C}$ for $45 \mathrm{~s}, 57^{\circ} \mathrm{C}$ for $30 \mathrm{~s}$, and $72^{\circ} \mathrm{C}$ for $30 \mathrm{~s}$. The genotype was estimated from the size of the PCR product analyzed by electrophoresis on $6 \%$ acrylamide gels stained with ethidium bromide.

\subsection{ELISA methods}

We used a novel and patented DAT-EIA-kit (patent holder: ISS, Italy), based on the ELISA method, most commonly used for determination in the serum of anti-neuronal auto-antibodies 
(aAbs), exploiting peptide fragments of the human DAT sequence as antigens (see Adriani et al., 2012). Briefly, the concentration of DAT-aAbs is determined using modified synthetic peptides, corresponding to a fragment of DAT, as antigens. These peptide fragments were designed by selecting a 19-aminoacid portion of the DAT sequence based on the most immuno-reactive portion (Granstrem et al. 10-AUG-2012 provisional patent; 10-AUG-2013 International Patent Application No. PCT/EP2013/066845), and custom synthesized by one of authors (O.G.).

The DAT-fragment antigen was affinely pre-adsorbed to the microplate $(0.5 \mu \mathrm{g} / \mathrm{well})$. In a first incubation step $\left(1 \mathrm{~h}, 25^{\circ} \mathrm{C}\right)$, the serum samples (diluted 1:100) are applied $(80 \mu 1$ per each well) on the immuno-plate (Costar, USA): antibodies react with the solid phase bound antigen. After intensive washing (PBS with 0.05\% Tween 20, pH 7.4), the captured antibodies are detected using monoclonal murine antibodies (catalogue number A8667; Sigma Aldrich, USA) to human IgG (diluted $5 \mathrm{~mL} / \mathrm{L}$ in vehicle: albumine $0.5 \mathrm{~g} / \mathrm{L}$, PBS-Tween $205 \mathrm{~mL} / \mathrm{L}$, thimerosal $0.02 \mathrm{~g} / \mathrm{L}$, sodium azide $0.05 \mathrm{~g} / \mathrm{L}$, distilled water; Sigma Aldrich, USA) conjugated with horseradish peroxidase (HP). This secondary antibody (diluted 1:20,000) is added (80 $\mu 1$ per each well) and incubated $\left(1 \mathrm{~h}, 25^{\circ} \mathrm{C}\right)$. Immuno-complex is semi-quantitatively determined by HRP/TMB-detection reaction: the substrate solution, TMB (catalogue number 09743; Fluka, USA) is added (80 $\mu 1$ per each well). Stopping solution $(2 \mathrm{~N} \mathrm{HCl})$ is then added converting the color to yellow. The plate is scanned at $450 \mathrm{~nm}$ on a Microplate reader 3550 (Bio-Rad, USA). The intensity of the yellow color is directly proportional to the concentration of DAT-aAbs in the serum sample.

\subsection{Statistical Analysis}

The analyses of behavioral scales, of titers, and correlations were run within all recruited ADHD patients $(\mathrm{N}=46)$; after 16 were excluded a posteriori for recent fever / allergy, the analyses involved only genotyped ADHD children $(\mathrm{N}=30)$. Subjects segregated into two DAT genotype groups $(\mathrm{N}=15$ each): DAT 9/9 genotype were too few to be reliably used for statistic purposes: henceforth, DAT 9/9 and 9/10 were pooled and referred to as "subjects with at least one 9-repeat DAT allele" (collectively termed 9/x).

First, we investigated the role of factors such as "group" (NPT vs. PT patients), "genotype" (DAT 10/10 vs. 9/x), "time" (T0 vs. T1). The following variables were evaluated: 1) scores 
obtained in CGAS and in subscales of Conners'; 2) semi-quantitative assay for anti-DAT titers, through the optical density, obtained with DAT-EIA-kit's ELISA assay.

Second, we run a full correlation analysis between anti-DAT titers, resulting from the DATEIA-kit's ELISA assay, and scores obtained by patients (in CGAS values assigned by clinicians; in Conners' Scales, compiled by parents and by teacher of the recruited children).

Third, we investigated the anti-DAT titers once again (time T2), after a 18-month period of exposure to MPH or to unmedicated conditions (NPT vs. PT patients, respectively), and compared them to the healthy controls (only DAT 9/x genotype, final $n=8$ ).

\section{RESULTS}

As outlined in Methods, we enrolled 46 ADHD subjects and 15 healthy controls; we analyzed aAbs titers and psychometric scales of all ADHD subjects; after a posteriori exclusion, we came to DAT genotype of 30 ADHD subjects (15 subjects per genotype), and eight healthy controls of DAT 9/x genotype. In general, the titer of DAT-aAbs was detectable (optical density around 0.81 in average) in all healthy controls and ADHD children, with a consistent elevation (optical density more than 1-1.1) in specific cases. As a whole, we report slightly but consistently elevated levels of DAT aAbs in the most severe ADHD patients; thus, determination of serum anti-DAT titers can be proposed as a suitable biomarker to help ADHD diagnosis.

\subsection{Symptom scales: CGAS and Conners'}

The average values of symptom scales filled in by the mothers and of CGAS values assigned by the clinician show, between time T0 and T1, a globally stable profile of ADHD symptoms in the group of children under non pharmacological therapy (NPT, 32 subjects before the exclusion made a posteriori), as illustrated in Fig. 1 upper panel. The Student t-test analysis did not yield any significant results hence suggesting no improvement in the absence of medication.

In the group of children placed under pharmacological treatment (PT, 14 subjects before the exclusion made a posteriori), the average values of symptoms (scales filled in by their mothers and CGAS values assigned by the clinician) showed clearly more severe ADHD symptoms, compared 
to those subjects who did not need to take a pharmacological therapy. As expected, a period of 6 weeks under MPH treatment led to an important reduction of ADHD symptoms between T0 and T1: the reduction of symptoms was significant for CGAS $(t(26)=3,67)$, for the attention deficit sub-scale ( $\mathrm{t}(26)=3,13)$, and for the ADHD index ( $(26)=3,27)$, as the Student $t$-test analysis demonstrated (Fig. 1 lower panel).

Figure 1 here

The Student t-test analysis that compared Conners' scales and CGAS values between the two groups (NPT vs PT subjects) revealed:

- Time T0: a significant difference in score for CGAS values $(\mathrm{t}(44)=3,79)$, for the attention deficit sub-scale $(\mathrm{t}(44)=2,92)$ and for the ADHD index $(\mathrm{t}(44)=2,10)$ : this finding suggests that the subjects, later assigned to the MPH treatment group, were characterized indeed by more severe ADHD symptoms compared to subjects who did not need to take a pharmacological therapy.

- Time T1: no significant differences were evidenced. This indicates that, after 6 weeks of pharmacological treatment, these subjects showed an important reduction in symptoms, so that they could no longer be distinguished from subjects who did not take any pharmacological therapy. The recovery of symptoms by MPH lead to children who appeared similar to unmedicated ones.

\subsubsection{Role of DAT genotype}

The group of subjects who did not take pharmacological therapy ( 9 were excluded for recent fever / allergy; 23 were genotyped out of 32 total) was split into two subgroups, namely in carriers of a DAT 10/10 (12 subjects) vs 9/x (11 subjects) genotype. It could be clearly observed that:

- Genotype 10/10 ( $n=12)$ : ADHD symptoms were unchanged from time T0 to T1 (Fig. 2 upper panel), suggesting no improvement at all following a non pharmacological intervention.

- Genotype 9/x (n=11): these subjects showed a more severe ADHD symptomatology at time T0, when compared to those carrying a 10/10 genotype $(t(21)=2,39)$. At time $T 1$, these subjects showed a slight reduction of all symptoms (possibly, due to parent counceling and/or behavioral therapy); however, the oppositional behavior was still more severe compared to subjects carrying a 10/10 genotype (Fig. 2 lower panel). Compared to 10/10 patients, the DAT 9/x ones could be suggested to be more prone to recovery in general, even without medication. 
Figures 2 and 3 here

Among subjects who needed to take pharmacological treatment, 7 were excluded and 7 were genotyped (out of 14 total). It is of note that (out of 7 excluded patients in the PT group) two were non responder to MPH and switched to atomoxetine, while five had recent fever / allergy. Then, this group was split into two subgroups, namely, carriers of DAT 10/10 (3 subjects: Fig. 3 upper panel) vs DAT 9/x (4 subjects: Fig. 3 lower panel) genotype. We observed that:

- Genotype 10/10 (n=3): subjects with both 10-repeat alleles showed just a trend towards a MPH-induced reduction of ADHD symptoms, according to CGAS and Conners' scales values, between T0 and T1; this reduction was however slight and did not reach statistical significance.

- Genotype 9/x (n=4): subjects with at least a DAT 9-repeat allele showed symptoms of inattention, hyperactivity and ADHD Index which were slightly more severe than those found in subjects with a 10/10 genotype, at time T0. These subjects, however, showed a better therapeutical response to MPH: in fact, a significant reduction of CGAS $(\mathrm{t}(6)=2,80)$ and of attention deficit Conners' sub-scale $(\mathrm{t}(6)=2,97)$ was demonstrated by the Student t-test analysis.

\subsection{Anti-DAT titer in plasma samples.}

The average aAbs titers in subjects who did not take pharmacological therapy showed a slight trend towards an increase in values from time T0 to T1 (Fig. 4 upper panel). This unexpected rise could represent a "physiological" phenomenon: at time T0, the sample was taken early in the morning (along with a routine blood screen), and could be therefore representative of the basal DAT aAbs titer; conversely, the sample withdrawn at time T1 could be used to estimate the "physiological" levels, reached in the late morning (and/or after having had a breakfast).

Figure 4 here

The average aAbs titer in subjects under pharmacological treatment showed, between time T0 and T1 time, a quite stable value (Fig. 4 lower panel). In these subjects, after 6 weeks of MPH treatment, we could not find an increasing titer, as we could have expected based on the findings from the non pharmacological therapy (NPT group). Noteworthy, at T1 time, patients were not taking the morning dose of the drug, therefore any differences between PT and NPT subjects can be considered as indicative of subchronic (and not acute) drug effect. 
When comparing the two groups (PT vs NPT), at time T0, we can observe that subjects, later to be assigned to pharmacological treatment, presented higher basal anti-DAT titers compared to those subjects later assigned to a non pharmacological therapy. These data allow us to hypothesize elevated basal anti-DAT titers in association with a more severe type of ADHD. Indeed, those PT patients were actually in need of a pharmacological approach. On the other hand, at time T1, this difference was no longer evident, because aAbs titers appeared to be similar in the two groups. Noteworthy, the analysis of behavioral profiles also evidenced very similar score between the two groups (Fig. 1). Thus, a close relationship between changes in behavior and in the immune marker apparently emerges. Indeed, PT and NPT subjects with similar aAbs titers (at T1, i.e. after 6 weeks of $\mathrm{MPH}$ or unmedicated conditions, respectively) also showed an indistinguishable behavioral profile. Notably, in the very same subjects, DAT aAbs titers did differ quite clearly when the symptoms also differed (i.e. at time T0). Thus, serum DAT aAbs titers could represent a valid and useful tool to monitor, among others, the efficacy of MPH.

\subsubsection{Role of DAT genotype}

By splitting the group of milder ADHD subjects, who did not need to take pharmacological therapy (NPT), into the two different DAT genotypes, it could be observed that:

- Time T0: basal aAbs titers did not differ for subjects carrying a 10/10 genotype (Fig. 5a) when compared to $9 / \mathrm{x}$ subjects (Fig. 5b);

- Time T1: subjects carrying a 9/x genotype showed a significant elevation of anti-DAT titers

from time $\mathrm{T} 0$ to $\mathrm{T} 1(\mathrm{t}(20)=2,01)$; however, at time $\mathrm{T} 1$, aAbs titers in subjects carrying a $9 / \mathrm{x}$ genotype didn't significantly differ from T1 titers in patients with a 10/10 genotype $(\mathrm{t}(21)=0.67)$. As such, a slight trend to increase can be proposed for 10/10 patients as well. By splitting the group of severe ADHD subjects, placed under MPH treatment (PT group), into the two different DAT genotypes, it can be observed that:

- Time T0: basal DAT aAbs titers of DAT 10/10 subjects (Fig. 5c) were almost double when compared to basal titers of $9 / \mathrm{x}$ subjects (Fig 5d). It is interesting to note that subjects, with severe symptoms and DAT 10/10 genotype, could be characterized by very high basal anti-DAT titers. 
- Time T1: for the 10/10 genotype, the aAbs titer was significantly increased when compared to that of $9 / x$ subjects $(t(5)=2,59)$. For the DAT 9/x genotype, there were no significant differences when the $\mathrm{T} 1$ titer was compared to the basal $\mathrm{T} 0$ titer. In conclusion, a high and drug-resistant titer (both basal and after treatment) may occur in DAT 10/10 people, i.e. the ones that also show a lower drug response as far as behavior is concerned (Fig. 3 upper panel). Conversely, while the titer apparently rises from T0 to T1 (in NPT patients), the aAbs titers are surprisingly not followed by such an increase in the group under MPH treatment, carrying a DAT 9/x genotype.

Figure 5 here

\subsection{Correlation between behavioral scales and DAT-aAbs.}

Anti-DAT titers and the behavioral scales filled in by the parents at T0 or T1 appeared significantly correlated in subjects under non pharmacological therapy. A significant correlation was found between titers, both at time T1 and basal, and the values of inattention indicated in questionnaires compiled by fathers; also, a significant trend was found between basal titers and hyperactivity values indicated in questionnaires compiled by teachers. These correlation values are illustrated in Table 1. This profile of data seems to indicate that, when the inattention levels (indicated in questionnaires compiled by the fathers and/or the motor behavior suggested by the teachers) do increase, also the DAT-aAbs circulating in the blood show an increase.

\section{Tables 1 and 2 here}

When considering the role of genotype in the NPT group, the observed correlation between aAbs titers and the attention disorder (i.e. the score in questionnaires compiled by the father) disappears for the $9 / \mathrm{x}$ subgroup. On the other hand, for the 10/10 subgroup, the correlations were confirmed and extended. A statistically significant correlation emerged between titers (both basal and at time T1) and all the behavioral scales, indicated in questionnaires compiled by fathers. Moreover, it can be found a significant relationship between anti-DAT titers (both basal and at time T1) and the oppositional behavior indicated in questionnaires compiled by the teacher (Table 2).

Correlations between DAT-aAbs and behavioral scales for subjects under pharmacological treatment (PT group) did not show any statistically significant correlation (data not shown). 


\subsection{Summary and comparison with control healthy subjects.}

Considering the role of genotype in the comparison between the two groups, we observe that:

1) Among 15 subjects carrying DAT 10/10 genotype, those three most severe cases, placed under a pharmacological treatment, turned out to show a doubled titer when compared to those 12 less severe cases, that were not in the need of a pharmacological therapy: this, both at time T0 (basal titer) and significantly at time $\mathrm{T} 1(\mathrm{t}(13)=2,06)$ after 6 weeks of therapy;

2) Subjects with a DAT $9 / x$ genotype did not show any difference in basal titer (time T0) between the two groups; in the T1 serum sample, measure of aAbs titers revealed a significant "physiological" increase in the 11 NPT subjects (Fig. 5b). Notably, no such elevation was found in the 4 PT patients. Indeed, a rise in titers was prevented by a 6-week MPH treatment.

The recruitment of 15 healthy controls, carried out around 18 months after the main study (at the time of patients' re-sampling, time T2), provided reliable samples from 8 subjects, who were

carrying at least one 9-repeat allele and not excluded a posteriori. These data were compared with eight 9/x patients, re-sampled (at the same time) after one-to-two years (time T2) of exposure to MPH or to the unmedicated condition (PT or NPT respectively). Interestingly, the control group presented a DAT aAbs titer of $0.838 \pm 0.178$ in average, a median of 0.601 and a third quartile of 1.027; the unmedicated ADHD children had a DAT aAbs titer of $1.673 \pm 0.201$ in average, with a median of 1.758 and a third quartile of 2,162; finally, the patients with 18-month long, daily exposure to MPH had a titer of $1.095 \pm 0.188$, with a median of 1.001 and a third quartile of 1.266.

The sample of unmedicated, 9/x ADHD children showed elevated titers compared to controls $(t(14) ; p=0.004)$; conversely, the 9/x ADHD children under MPH treatment showed significantly reduced DAT aAbs titers $(\mathrm{t}(14) ; \mathrm{p}=0.029)$, which were hardly distinguishable from those of controls. This latter finding, collected from eight re-sampled patients, can be compared with findings already collected from the very same 9/x children at T1: indeed, a 6-week MPH exposure (PT group, see Fig. 5b) was already found to prevent the rise of T1 titers, otherwise shown by the unmedicated subjects (NPT group, see Fig. 5d). As a whole, a main suggestion seems to emerge from these data: the majority of recruited ADHD children showed DAT aAbs titers (between 0.4 and 0.9 ) fully comparable to those of healthy subjects, carrying a 9/x genotype. 
Interestingly, specific profiles emerged as a function of the genotype. The 10/10 ADHD children, in the three most severe cases needing drug treatment, showed an elevated titer (almost doubled, and much more than 1-1.1); also, in 12 less severe cases, the titer turned out to correlate quite well with symptoms' severity. Their DAT aAbs titer resulted, however, not sensitive to MPH exposure, and these subjects also did not show reliable relief of behavioral symptoms either. The 9/x ADHD children showed - conversely - a quite interesting similarity between serum titers and behavioral symptoms, in that both appeared sensitive to MPH. Noteworthy, the 4 most severe cases showed relief of symptoms after just 6 weeks of therapy and, furthermore, the elevation of titers (expected based on all NPT-group findings) was prevented if subjects were medicated; this, both after 6 weeks (time T1) and following a longer, 1-to-2 year period (time T2). However, the aAbs titer did not correlate with individual symptoms' severity, neither before nor after medication.

\section{DISCUSSION}

Nowadays, the diagnosis of ADHD is mainly based on structured interviews, questionnaires, and physicians' judgment following clinical observation. Nevertheless, the diagnosis of ADHD is not unequivocal; indeed, the behavioral nature of these criteria renders ADHD diagnosis yet a debated question, at least in borderline conditions. There is currently much interest in the search for new biological and measurable markers, possibly detectable via a simple biochemical tool and with non-invasive, peripheral approaches (Scassellati et al., 2012).

The clinical-assessment data from subjects in the NPT group have shown a globally stable profile of ADHD symptoms over time (i.e. between recruitment and follow-up, at 6 weeks after recruitment). This stable profile was particularly evident for patients with DAT 10/10 genotype. On the other hand, subjects later assigned to a pharmacological treatment (PT) showed, at time of recruitment, more severe ADHD symptoms compared to NPT subjects, confirming that PT patients were actually in the need to take a pharmacological therapy. Furthermore, after 6 weeks under MPH treatment, some subjects showed an important and significant reduction in ADHD symptoms. By considering DAT genotype, we found that a reduction of ADHD symptoms over time (between recruitment and follow-up, 6 weeks after MPH reached the therapeutic dosage) was marked (and 
statistically significant) only in the subgroup of subjects with a DAT 9/x genotype. This confirms a greater efficacy of pharmacological treatment, in reducing ADHD symptoms, for patients bearing at least one 9-repeat DAT allele.

Our primary aim was to measure the blood levels of auto-antibodies (aAbs) targeting epitopes of the DAT protein. Interestingly, all children (including healthy controls) revealed measurable levels of DAT-directed aAbs. Notably, among patients, specific subjects showed an elevated titer. Moreover, as a function of genotype, titers apparently depended on behavioral symptoms and/or on MPH exposure. A first notion is that severe ADHD subjects - which were assigned to MPH pharmacological treatment - presented, at recruitment (time T0), higher anti-DAT titers compared to the mild ADHD group - which was assigned to a non pharmacological therapy. These data suggest an association between elevated basal anti-DAT titers and a more severe profile of ADHD symptoms, therefore identifying the need of a pharmacological approach.

A second notion is that levels of these aAbs were not stable in unmedicated conditions. Subjects who entered a non pharmacological therapy showed increasing titers from time $\mathrm{T} 0$ to $\mathrm{T} 1$, and further elevation when re-sampled 1-to-2 years later. This unexpected rise could represent, possibly, a "patho-physiological" phenomenon. It should be noted that NPT children were left in unmedicated conditions between $\mathrm{T} 1$ and $\mathrm{T} 2$, leaving room for the hypothesis that anti-DAT titers may indicate a progressively deteriorating immunological condition. We cannot however exclude the existence of a circadian physiological fluctuation in the aAbs titer, with increasing levels of circulating aAbs in the late morning and/or after food intake.

After 6 weeks from recruitment, overlapping behavioral profiles and similar aAbs titers were observed between subjects exposed to unmedicated conditions vs MPH treatment. Noteworthy, these two groups were different at the time of recruitment (i.e. PT patients showed elevated aAbs titer and more severe symptoms' profile). After 6 weeks from recruitment, children placed under pharmacological treatment did not show the rise in T1 aAbs titer over T0 baseline, conversely observed for NPT patients. In the PT subjects, the subchronic exposure to MPH treatment was apparently preventing the increase in anti-DAT titer. This information can lead us to propose, therefore, that anti-DAT titers could be used to monitor the efficacy of MPH. 


\subsection{Role of DAT genotype.}

By splitting the whole PT and NPT subjects into two subgroups, according to DAT genotype, we could observe that only subjects carrying a 9/x genotype (and under non pharmacological therapy, NPT) showed indeed the aforementioned, statistically significant increase of anti-DAT titers from $\mathrm{T} 0$ baseline to time T1. This inerease from the PT and possibly "patho-physiological" increase over T0 baseline. This profile, however, was only evident in the group of NPT subjects, and notably no such an increase was found due to a 6-week MPH treatment. We suggest that, in DAT 9/x subjects placed under pharmacological treatment, a subchronic exposure to MPH prevented the increase of aAbs titers between $\mathrm{T} 0$ and $\mathrm{T} 1$, otherwise observed for subjects carrying a DAT 9/x genotype but not placed under pharmacological therapy. It is tempting to speculate that therapeutic effects of MPH might be accompanied by a specific modulation (i.e. preventing the spontaneous increase) of DAT aAbs titers.

In the group that needed to undergo MPH treatment, subjects with a DAT 10/10 genotype showed a very high levels of DAT-aAbs: the anti-DAT titers were almost double when compared both to those of DAT 9/x subjects, and to those of less severe DAT 10/10 patients, not placed under MPH. Anti-DAT levels were higher at baseline and further elevated after 6-week pharmacological treatment, accompanied by the very slight and not significant reduction in symptoms' severity, reported by the clinician and parents. This suggests that subjects with a DAT 10/10 genotype could be characterized by a lower therapeutic response to MPH treatment (Madras et al. 2002). It is tempting to speculate that reduced drug efficacy comes along with lack of any effect over the very high (and rising) anti-DAT titer. This may suggests that, in case of ADHD subjects who are homozygous for DAT 10-repeat alleles, high and drug-resistant DAT aAbs titers can identify those patients in need of alternative, non psycho-stimulant drug treatment (like e.g. atomoxetine).

\subsection{Behavioral scales / anti-DAT titer correlations.}

The analysis of correlations was run between behavioral scales (filled in by the clinician, the parents and the teacher) and DAT aAbs titers. A profile of correlation was found in subjects under a non pharmacological therapy. A significant correlation was found between basal aAbs titers and 
the values of inattention indicated by fathers; also, a significant trend was found between basal aAbs titers and hyperactivity values as indicated by patient's teacher. This means that, when inattention level suggested by fathers and/or the motor behavior suggested by teachers is increased, the circulating DAT-aAbs may turn out to be elevated.

By splitting NPT patients into two subgroups, according to DAT genotype, the above correlations were confirmed and further extended for the DAT 10/10 subgroup. A statistically significant correlation emerged between titers (both basal and at time T1) and al/ the behavioral scales suggested by fathers. Moreover, we could find a significant relationship between titers (both basal and at time T1) and oppositional behavior indicated by the teacher. Therefore, in homozygous subjects carrying the DAT 10-repeat allele, the highest levels of DAT-aAbs came along with the more problematic behavior of children. Consistently, the most severe ADHD cases, in need of drug treatment (PT group), showed almost doubled DAT aAbs titers (see above). On the other hand, the correlation between scales and titers completely disappeared for DAT 9/x subgroup.

\subsection{Patho-physiological remarks and perspectives.}

Abnormal dopamine levels and deregulation of dopaminergic components, expressed in immune cells, are associated with some autoimmune disorders (Pacheco et al., 2014). Dopamine can probably activate T-cell function indirectly, by suppressing T-regulatory cell (Treg) (Cosentino et al., 2007; Kipnis et al., 2004; Levite, 2008). Treg is involved in autoimmunity, and secretes IL10 and TGF-b: because of its inhibitory properties, reduced activity of Treg (due to abnormal dopamine) could lead to uncontrolled activation and function of effector T-cells and to autoimmunity (Pacheco et al., 2010). Furthermore, dendritic-cell derived dopamine promotes IL-23 production and, thereby, enhances Th17 responses with inflammation (Pacheco et al. 2014). These findings suggest that dopamine can mediate communication between immune cells, and the crosstalk between the immune and nervous systems (Pacheco et al., 2014; Buttarelli et al., 2011; Nakano et al., 2009; Cosentino et al., 2007; Sarkar et al., 2010).

Thus, over-expression of DAT in lymphocytes of ADHD children could imply dysregulation of immune and neuro-immune systems. A reduced level of dopamine is typical of autoimmune pathologies (Buttarelli et al., 2011). Blockade of DAT by MPH may normalize the dopamine 
levels, which in turn may act on the lymphocytes. It was reported, consistently, that MPH may induce (i) a hyperactivity of the immune system and a heightened tendency to respond to antigenic or immunogenic stimuli, (ii) hyper-gamma-globulinemia, and (iii) reduction of T-helper/inducer cells (Auci et al., 1997). The issue of a role for neuro-immune components in ADHD and other developmental, neuro-psychiatric conditions deserves however further investigation.

\subsection{Conclusion.}

Replication of our findings is needed: it will be necessary to investigate the selectivity of epitopes (i.e. to ascertain that aAbs recognize epitopes of DAT over other proteins), as well as their specificity (i.e. how do these DAT aAbs vary in other mental or immunological conditions which are not related to ADHD). For validation of DAT aAbs as a tool for diagnosis and/or therapy monitoring in subjects with ADHD, we would need to clarify the aetiological implications: namely, the nature of the correlation between anti-DAT titers, on one hand, and clinical features of ADHDlike behavioral symptoms, on the other hand.

Present data do indicate for the first time that detectable levels of a DAT-directed, circulating auto-antibody can be evidenced in a pediatric population, including children with ADHD diagnosis. In addition, we propose that anti-DAT titers and DAT genotyping might possibly be used together, to determine a priority for drug intervention based on severity of child's symptoms. In other words, the elevation of serum anti-DAT titers and restoration of basal levels by few weeks of MPH can be proposed as bio-markers, to support diagnosed ADHD and its drug therapy. These biological indices could turn out to be useful: 1) to provide or verify diagnostic criteria, 2) to predict the evolution of symptoms over time, and 3) to monitor the efficacy of MPH treatment.

In the end, further study is warranted to deepen the knowledge about ADHD neurobiology, by exploring the relationship between this neuro-psychiatric disorder and the immune system. The presence of elevated circulating aAbs directed against DAT in ADHD subjects may well open new avenues of (pre)clinical research, as neuro-immune modulations are increasingly worthy of being explored. 


\section{TABLES, LEGENDS}

Table 1. Values of Pearson's R in correlations run between behavioral scores (obtained in CGAS and in various Conners' subscales compiled by parents and teachers, see Columns) and the DAT-aAbs titers, for children assigned to the non-pharmacology therapy (NPT) group. Data include also ADHD children which were then not genotyped due to recent fever / allergy $(\mathrm{N}=32)$. First line: behavioral scores at recruitment correlated to baseline titers (at T0); Second line: behavioral scores at 6-week follow-up correlated to corresponding titers (at T1); Third line: behavioral scores at recruitment correlated to 6-week follow-up titers (at T1). The few cases where correlation is significant are for $\mathrm{R}>0.34(\mathrm{~N}=32)$.

\begin{tabular}{|c|c|c|c|c|c|c|c|c|c|c|c|c|c|}
\hline & \multicolumn{13}{|c|}{$\begin{array}{l}\text { NPT } \\
\end{array}$} \\
\hline & \multicolumn{5}{|c|}{ Conners' mother } & \multicolumn{4}{|c|}{ Conners' father } & \multicolumn{4}{|c|}{ Conners' teacher } \\
\hline & CGAS & oppos.behav. & attention deficit & hyperactivity & AADHDI & oppos.behav. & attention deficit & hyperactivity & ADHD & oppos.behav. & attention deficit & hyperactivity & JADHDT \\
\hline average T0 & 0,091 & $-0,056$ & 0,158 & $-0,122$ & $-0,007$ & 0,106 & 0,514 & 0,133 & 0,269 & 0,151 & 0,253 & 0,329 & 0,295 \\
\hline average $\mathrm{T1}$ & 0,057 & 0,102 & 0,047 & 0,013 & 0,048 & 0,078 & 0,010 & $-0,042$ & 0,021 & & & & \\
\hline ct0vst1 & 0,032 & 0,005 & 0,123 & $-0,004$ & 0,016 & 0,176 & 0,419 & 0,202 & 0,274 & 0,093 & 0,123 & 0,246 & 0,185 \\
\hline
\end{tabular}

Table 1.

Table 2. Same as in Table 1, but divided for genotype DAT 10/10 (panel a, $n=12$ ) vs. DAT 9/x (panel $b, n=11$ ). Teachers' evaluation was collected only at recruitment and not at 6-week follow-up. Interestingly, in DAT 10/10 patients at risk for ADHD, higher DAT-aAbs titers are related with poorer assessment by Conners' scales. Correlation is significant for $\mathrm{R}>0.53(\mathrm{n}=12)$.

\begin{tabular}{|c|c|c|c|c|c|c|c|c|c|c|c|c|c|}
\hline & \multicolumn{13}{|c|}{ NPT: genotipe DAT 10/10 } \\
\hline & & \multicolumn{4}{|c|}{ Conners' mother } & \multicolumn{4}{|c|}{ Conners' father } & \multicolumn{4}{|c|}{ Conners' teacher } \\
\hline & CGAS & oppos.behav. & attention deficit & hyperactivity & ADHD & oppos.behav. & attention deficit & Thyperactivity & ADHD & oppos.behav. & attention deficit & Thyperactivity & ADHDT \\
\hline average T0 & $-0,349$ & 0,237 & 0,374 & $-0,150$ & $-0,212$ & 0,610 & 0,740 & 0,545 & 0,543 & 0,578 & 0,302 & 0,400 & 0,399 \\
\hline average $\mathrm{T1}$ & $-0,002$ & 0,184 & $-0,157$ & $-0,243$ & $-0,338$ & 0,193 & $-0,157$ & $-0,243$ & $-0,355$ & & & & \\
\hline c tovs t1 & $-0,263$ & 0,112 & 0,103 & $-0,131$ & $-0,404$ & 0,434 & 0,548 & 0,457 & 0,325 & 0,503 & 0,324 & 0,448 & 0,419 \\
\hline & \multicolumn{13}{|c|}{ NPT: genotipe DAT $9 / x$} \\
\hline & & \multicolumn{4}{|c|}{ Conners' mother } & \multicolumn{4}{|c|}{ Conners' father } & \multicolumn{4}{|c|}{ Conners' teacher } \\
\hline & CGAS & oppos.behav. & attention deficit & hyperactivity & ADHDI & oppos.behav. & attention deficit & hyperactivity & ADHD & oppos.behav. & attention deficit & hyperactivity & ADHDT \\
\hline average T0 & 0,179 & $-0,300$ & 0,233 & $-0,261$ & 0,090 & $-0,428$ & 0,267 & $-0,117$ & 0,020 & $-0,154$ & 0,895 & 0,070 & 0,351 \\
\hline average $\mathrm{T1}$ & $-0,225$ & 0,103 & 0,315 & 0,261 & 0,286 & $-0,129$ & 0,120 & 0,033 & 0,082 & & & & \\
\hline c tovst1 & $-0,155$ & $-0,154$ & 0,230 & 0,173 & 0,188 & $-0,084$ & 0,232 & 0,171 & 0,233 & $-0,533$ & 0,490 & $-0,270$ & $-0,097$ \\
\hline
\end{tabular}

Table 2, panels a) and b). 


\section{REFERENCES}

Achenbach, T.M. Manual for the child behavior checklist / 4-18 and 1991 profile. University of Vermont, Burlington VT, USA (1991).

Adriani, W., Koot, S., Columba-Cabezas, S., Romano, E., Travaglini, D., van den Bos, R., Granstrem, O., Ali, S.F., \& Laviola, G. Immunization with DAT fragments is associated with long-term striatal impairment, hyper-activity and reduced cognitive flexibility in mice. Behav Brain Funct 8, 54 (2012).

American Psychiatric Association. Diagnostic and statistical manual of mental disorders. $4^{\text {th }}$ Ed.. Text Revision. Washington DC, USA (2000).

Ankeny, D.P. \& Popovich, P.G. B cell and autoantibodies: complex roles in CNS injury. Trends Immuno/31, 332-8 (2010).

Auci, D.L., Fikrig, S., Rodriquez, J. Methylphenidate and the immune system. J Am Acad Child Adolesc Psychiatry 36, 1015-6 (1997)

Bannon, M.J. The dopamine transporter: role in neurotoxicity and human disease. Toxicol App/ Pharmacol 204, 355-60 (2005).

Berridge, K.C. The debate over dopamine's role in reward: the case for incentive salience. Psychopharmacology 191, 391-431 (2007).

Biderman, J., Monuteaux, M.C., Mick, E., Spencer, T., Wilens, T.E., Silva, J.M., Snyder, L.E. \& Faraone, S.V. Young adult outcome of attention deficit hyperactivity disorder: a controlled 10-year followup study. Psychol Med 36, 167-79 (2006).

Buttarelli, F.R., Fanciulli, A., Pellicano, C., Pontieri, F.E. The dopaminergic system in peripheral blood lymphocytes: from physiology to pharmacology and potential applications to neuropsychiatric disorders. Curr Neuropharmaco/9, 278-88 (2011)

Capone, F. , Adriani, W. , Shumilina, M., Izykenova, G., Granstrem, O., Dambinova, S., Laviola, G. Auto-antibodies against opioid or glutamate receptors are associated with changes in morphine reward and physical dependence in mice. Psychopharmacology 197, 535-48 (2008).

Chambers, R.A. \& Potenza, M.N. Neurodevelopment, impulsivity, and adolescent gambling. J Gamb/ Stud 19, 53-84 (2003). 
Chong, J,Y, Rowland, L.P. \& Utiger, R.D. Hashimoto encephalopathy: syndrome or myth? Arch Neurol60, 164-71 (2003).

Cohen-Solal, J.F. \& Diamond, B. Neuropsychiatric lupus and autoantibodies against ionotropic glutamate receptor (NMDAR). Rev Med Intern 32, 130-2 (2011).

Colasanti, T., Delunardo, F., Margutti, P., Vacirca, D., Piro, E., Siracusano, A. \& Ortona, E. Autoantibodies involved in neuropsychiatric manifestations associated with systemic lupus erythematosus. $J$ Neuroimmuno/212, 3-9 (2009).

Conners, C.K., Sitarenios, G., Parker, J.D., \& Epstein, J.N. The revised Conners' Parent Rating Scale (CPRS-R): Factor structure, reliability, and criterion validity. J Abnorm Child Psycho/26, 257-68 (1998).

Cook, E.H. Jr, Stein, M.A., Krasowski, M.D., Cox, N.J., Olkon, D.M., Kieffer, J.E., Leventhal, B.L. Association of attention deficit hyperactivity disorder and the dopamine transporter gene. Am J Hum Genet 56, 993-998 (1995)

Cortese, S. The neurobiology and genetics of Attention-Deficit/Hyperactivity Disorder (ADHD): What every clinician should know. Eur J Paediatr Neurol 16, 422-33 (2012).

Cosentino, M., Fietta, A.M., Ferrari, M., Rasini, E., Bombelli, R., Carcano, E., Saporiti, F., Meloni, F., Marino, F., Lecchini, S. Human CD4+CD25+ regulatory T cells selectively express tyrosine hydroxylase and contain endogenous catecholamines subserving an autocrine/paracrine inhibitory functional loop. Blood $109,632-42(2007)$

Curatolo, P., Paloscia, C., D’Agati, E., Moavero, R. \& Pasini, A. The neurobiology of attention deficit/hyperactivity disorder. Eur J Paediatr Neurol 13, 299-304 (2009).

Dalmau, J., Lancaster, E., Martinez-Hernandez, E., Rosenfeld, M.R. \& Balice-Gordon, R. Clinical experience and laboratory investigations in patients with anti-NMDAR encephalitis. Lancet Neuro/ 10, 6374 (2011).

Dambinova, S.A., Granstrem, O., Tourov, A., Salluzzo, R., Castello, F. \& Izykenova, G.A. Monitoring brain spiking activity and autoantibodies to N-terminus domain of GluR1 subunit of AMPA receptors in blood serum of rats with cobalt-induced epilepsy. J Neurochem 71, 2088-93 (1998).

Dambinova, S.A., Izykenova, G.A., Burov, S.V., Grigorenko, E.V. \& Gromov, S.A. The presence of autoantibodies to N-terminus domain of GluR1 subunit of AMPA receptor in the blood serum of patients with epilepsy. J Neurol Sci 152, 93-7 (1997). 
Dambinova, S.A., Khounteev, G.A., Izykenova, G.A., Zavolokov, I.G., Ilyukhina, A.Y. \& Skoromets, A.A. Blood test detecting autoantibodies to NMDA neuroreceptors for evaluation of patients with transient ischemic attack and stroke. Clin Chem 49, 1752-62 (2003).

Davies, A.L., Hayes, K.C. \& Dekaban, G.A. Clinical correlates of elevated serum concentrations of cytokines and autoantibodies in patients with spinal cord injury. Arch Phys Med Rehabil 88, 1384-93 (2007).

Davis, C.C., Claudiud, M., Palinkas, L.A., Wong, J.B. \& Leslie, L.K. Putting families in the center: family perspectives on decision making and ADHD and implications for ADHD care. J Atten Disord 16, 675-84 (2011).

Diamond, B., Huerta, P.T., Mina-osorio, P., Kowal, C. \& Volpe, B.T. Losing your nerves? Maybe it's the antibodies. Nat Rev Immuno/ 9, 449-56 (2009).

Fontenelle, L.F., Oostermeijer, S., Harrison, B.J., Pantelis, C., \& Yücel, M. Obsessive-compulsive disorder, impulse control disorders and drug addiction: common features and potential treatments. Drugs 71 , $827-40(2011)$.

Gill, M., Daly, G., Heron, S., Hawi, Z., Fitzgerald, M. Confirmation of association between attention deficit hyperactivity disorder and a dopamine transporter polymorphism. Mol Psychiatry 2, 311-313 (1997)

Granstrem, O., Adriani, W., Shumilina, M., Izykenova, G., Dambinova, S. \& Laviola, G. Specific changes in levels of auto-antibodies to glutamate and opiate receptors induced by morphine administration in rats. Neurosci Lett 403, 1-5 (2006).

Graus, F., Boronat, A., Xifro, X., Boix, M., Svigeli, V., Garcia, A., Palomino, A., Sabater, L., Alberch, J. \& Saiz, A. The expanding clinical profile of anti-AMPA receptor encephalitis. Neurology 74, 857-9 (2010).

Graus, F., Saiz, A. \& Dalmau, J. Antibodies and neuronal autoimmune disorders of the CNS. J Neurol 257, 509-17 (2010).

Graus, F., Saiz, A., Lai, M., Bruna, J., López, F., Sabater, L., Blanco, Y., Rey, M.J., Ribalta, T. \& Dalmau, J. Neuronal surface antigen antibodies in limbic encephalitis: clinical-immunologic associations. Neurology 71, 930-6 (2008).

Hegvik, T.A., Husebye, E.S., \& Haavik, J. Autoantibodies targeting neurotransmitter biosynthetic enzymes in attention-deficit/hyperactivity disorder. Eur Child Adolesc Psychiatry. 23, 115-117 (2014). 
Hinshaw, S.P., Scheffler R.M., Fulton, B.D., Aase, H., Banaschewski, T., Cheng, W., Mattos, P., Holte, A., Levy, F., Sadeh, A., Sergeant, J.A., Taylor, E. \& Weiss, M.D. International variation in treatment procedures for ADHD: social context and recent trends. Psychiatr Serv62, 459-64 (2011).

Hoekstra, P.J. \& Minderaa, R.B. Tic disorders and obsessive-compulsive disorder: is autoimmunity involved? Int Rev Psychiatry 17, 497-502 (2005).

Hollander, E., Buchalter, A.J. \& DeCaria, C.M. Pathological gambling. Psychiatr Clin North Am 23, 629-42 (2000).

Hollander, E., Sood, E., Pallanti, S., Baldini-Rossi, N. \& Baker, B. Pharmacological treatments of pathological gambling. J Gamb/ Stud 21, 99-110 (2005).

Jucaite, A., Fernell, E., Halldin, C., Forssberg. H. \& Farde, L. Reduced midbrain dopamine transporter binding in male adolescents with attention-deficit/hyperactivity disorder: association between striatal dopamine markers and motor hyperactivity. Biol Psychiatry57, 229-38 (2005).

Kaufman, J., Birmaher, B., Brent, D., Rao, U., Flynn, C., Mareci, P., Williamson, D. \& Ryan, N. Schedule for Affective Disorders and Schizophrenia for School-Age Children-Present and Lifetime Version (K-SADS-PL): Initial reliability and validity data. J Am Acad Child Adolesc Psychiatry 36, 980-88 (1997).

Kipnis, J., Cardon, M., Avidan, H., Lewitus, G.M., Mordechay, S., Rolls, A., Shani, Y., Schwartz, M. Dopamine, through the extracellular signal-regulated kinase pathway, downregulates CD4+ CD25+ regulatory T-cell activity: implications for neurodegeneration. J Neurosci 24, 6133-43 (2004)

Knight, J.G., Menkes, D.B., Highton, J. \& Adams, D.D. Rationale for a trial of immuno-suppressive therapy in acute schizophrenia. Mol Psychiatry 12, 424-31 (2007).

Kowal, C., Degiorgio, L.A., Lee, J.Y., Edgar, M.A., Huerta, P.T., Volpe, B.T. \& Diamond, B. Human lupus autoantibodies against NMDA receptors mediate cognitive impairment. Proc Natl Acad Sci USA 103, 19854-9 (2006).

Kuang, F., Wang, B.R., Zhang, P., Fei, L.L., Jia, Y., Duan, X.L., Wang, X., Xu, Z., Li, G.L., Jiao, X.Y. \& Ju, G. Extravasation of blood-borne immunoglobulin $\mathrm{G}$ through blood-brain barrier during adrenaline-induced transient hypertension in the rat. Int J Neurosci 114, 575-91 (2004).

Lai, M., Hughes, E.G, Peng, X., Zhou, L, Gleichman, A.J., Shu, H., Matà, S., Kremens, D., Vitaliani, R., Geschwind, M.D., Bataller, L., Kalb, R.G., Davis, R., Graus, F., Lynch, D.R., Balica-Gordon, R. \& 
Dalmau, J. AMPA receptor antibodies in limbic encephalitis alter synaptic receptor location. Ann Neuro/65, 424-34 (2009).

Lancaster, E., Lai, M., Peng, X. Hunghes, E., Constantinescu, R., Raizer, J., Friedman, D., Skeen, M.B., Grisold, W., Kimura, A., Ohta, K., Iizuka, T., Guzman, M., Graus, F., Moss, S.J., Balice-Gordon, R. \& Dalmau, J. Antibodies to the GABA(B) receptor in limbic encephalitis with seizures: case series and characterisation of the antigen. Lancet Neuro/9, 67-76 (2010).

Levin, E.C., Acharya, N.K., Han, M., Zavareh, S.B., Sedeyn, J.C., Venkataraman, V. \& Nagele, R.G. Brain-reactive autoantibodies are nearly ubiquitous in human sera and may be linked to pathology in the context of blood-brain barrier breakdown. Brain Res 1345, 221-32 (2010).

Levite, M. Neurotransmitters activate T-cells and elicit crucial functions via neurotransmitter receptors. Curr Opin Pharmaco/8, 460-71 (2008)

Madras, B.K., Miller, G.M., Fischman, A.J. The dopamine transporter: relevance to attention deficit hyperactivity disorder (ADHD). Behav Brain Res 130, 57-63 (2002)

Martino, D., Dale, R.C., Gilbert, D.L., Giovannoni, G., \& Leckman, J.F. Immunopathogenic mechanisms in tourette syndrome: A critical review. Mov Disord 24, 1267-79 (2009)

Nakano, K., Higashi, T., Takagi, R., Hashimoto, K., Tanaka, Y., Matsushita, S. Dopamine released by dendritic cells polarizes Th2 differentiation. Int Immuno/21, 645-54 (2009).

Oades, R.D. Frontal, temporal and lateralized brain function in children with attention-deficit hyperactivity disorder: a psychophysiological and neuropsychological viewpoint on development. Behav Brain Res 94, 83-95 (1998).

Pacheco, R., Contreras, F., Zouali, M. The dopaminergic system in autoimmune diseases. Front Immuno/5, 117 (2014)

Pacheco, R., Riquelme, E., Kalergis, A.M. Emerging evidence for the role of neurotransmitters in the modulation of T cell responses to cognate ligands. Cent Nerv Syst Agents Med Chem 10, 65-83 (2010)

Passarelli, F., Donfrancesco, R., Nativio, P., Pascale, E., Di Trani, M., Patti, A.M., Vulcano, A., Gozzo, P., \& Villa, M.P. Anti-Purkinje cell antibody as a biological marker in attention deficit/hyperactivity disorder: a pilot study. J Neuroimmuno/ 258, 67-70 (2013)

Polanczyk, G., De Lima, M.S., Horta, B.L., Biederman, J. \& Rohde, L.A. The worldwide prevalence of ADHD: a systematic review and metaregression analysis. Am J Psychiatry 164, 942-8 (2007). 
Purper-Ouakil, D., Ramoz, N., Lepagnol-Bestel, A.M., Gorwood, P. \& Simonneau, M. Neurobiology of attention deficit/hyperactivity disorder. Pediatr Res 69, 69-76 (2011).

Rizzo, R. Gulisano, M., Calì, P.V. \& Curatolo, P. ADHD and epilepsy in children with Tourette syndrome: a triple comorbidity? Acta Paediatr 99, 1894-6 (2010).

Rogers, S.W., Andrews, P.I., Gahring, L.C., Whisenand, T., Cauley, K., Crain, B., Hughes, T.E., Heinemann, S.F. \& McNamara, J.O. Autoantibodies to glutamate receptor GluR3 in Rasmussen's encephalitis. Science 265, 648-51 (1994).

Sagvolden, T. \& Sergeant, J.A. Attention deficit/hyperactivity disorder-from brain dysfunction to behaviour. Behav Brain Res 94, 1-10 (1998).

Sano, A., Kondoh, K., Kakimoto, Y. \& Kondo, I. A 40-nucleotide repeat polymorphism in the human dopamine transporter gene. Hum Genet 91, 405-6 (1993).

Sarkar, C., Basu, B., Chakroborty, D., Dasgupta, P.S., Basu, S. The immunoregulatory role of dopamine: an update. Brain Behav Immun 24, 525-8 (2010)

Scassellati, C., Bonvicini, C., Faraone, S.V. \& Gennarelli, M. Biomarkers and attention-deficit / hyperactivity disorder: a systematic review and meta-analyses. J Am Acad Child Adolesc Psychiatry 51, 1003-19 (2012).

Seeman, P., \& Madras, B. Methylphenidate elevates resting dopamine which lowers the impulsetriggered release of dopamine: a hypothesis. Behav Brain Res. 130: 79-83 (2002).

Shaffer, D., Gould, M. S., Brasic, J., Ambrosini, P., Fischer, P., Bird, H. \& Aluwahlia, S. A children’s global assessment scale (CGAS). Arch Gen Psychiatry 40, 1228-31 (1983).

Sheppard, D.M., Bradshaw, J.L., Purcell, R. \& Pantelis, C. Tourette's and comorbid syndromes: obsessive compulsive and attention deficit hyperactivity disorder. A common etiology? Clin Psychol Rev19, 531-52 (1999).

Shulman, S.T. Pediatric autoimmune neuropsychiatric disorders associated with streptococci (PANDAS): update. Curr Opin Pediatr 21, 127-30 (2009).

Sonuga-Barke, E.J. Causal models of attention-deficit/hyperactivity disorder: from common simple deficits to multiple developmental pathways. Biol Psychiatry57, 1231-38 (2005). 
Swanson, J.M. The SNAP-IV Teacher and Parent Rating Scale. University of California, Irvine CA, USA (1983).

Teixeira, A.L., Rodrigues, D.H., Marques, A.H., Miguel, E.C., \& Fontenelle, L.F. Searching for the immune basis of obsessive-compulsive disorder. Neuroimmunomodulation 21, 152-8 (2014)

Twyman, R.E., Gahring, L.C., Spiess, J. \& Rogers, S.W. Glutamate receptor antibodies activate a subset of receptors and reveal an agonist binding site. Neuron 14, 755-62 (1995).

Vandenbergh, D.J., Persico, A.M. \& Uhl, G.R. A human dopamine transporter cDNA predicts reduced glycosylation, displays a novel repetitive element and provides racially-dimorphic TaqI RFLPs. Brain Res Mol Brain Res 15, 161-6 (1992).

Vincent, A., Lily, O. \& Palace, J. Pathogenic autoantibodies to neuronal proteins in neurological disorders. J Neuroimmunol 100, 169-80 (1999).

Waldman, I.D., Rowe, D.C., Abramowitz, A., Kozel, S.T., Mohr, J.H., Sherman, S.L., Cleveland, H.H., Sanders, M.L., Gard, J.M., Stever, C. Association and linkage of the dopamine transporter gene and attention-deficit hyperactivity disorder in children: heterogeneity owing to diagnostic subtype and severity. Am J Hum Genet 63, 1767-76 (1998)

Wechsler, D. Wechsler Scales of Intelligence (3rd Ed.; Italian Ed. of 2006). Psychological Corporation, San Antonio TX, USA (1991).

Zandi, M.S., Irani, S.R., Lang, B., Waters, P., Jones, P.B., McKenna, P., Coles, A.J., Vincent, A. \& Lennox, B.R. Disease-relevant autoantibodies in first episode schizophrenia. J Neuro/ 258, 686-8 (2010).

Zuliani, L., Graus, F., Giometto, B., Bien, C. \& Vincent, A. Central nervous system neuronal surface antibody associated syndrome: review and guidelines for recognition. J Neurol Neurosurg Psychiatry 83, 638-45 (2012). 

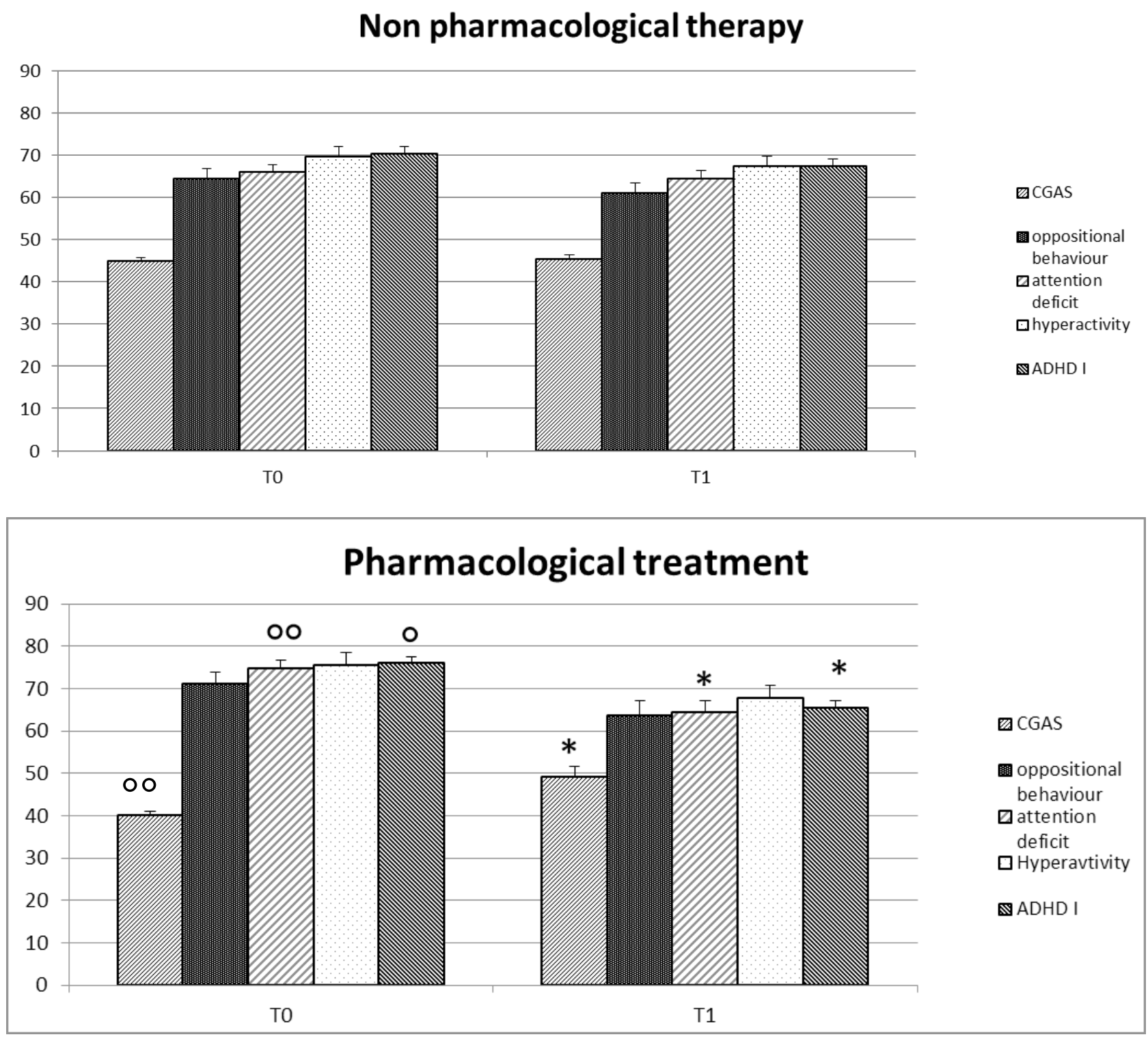

\section{Figure 1}

(upper panel) - Psychometric scales in recruited ADHD children not assigned to a pharmacotherapy (NPT, N=32). (lower panel) - Psychometric scales in recruited ADHD children assigned to pharmacological treatment $(\mathrm{PT}, \mathrm{N}=14)$. Data include also ADHD children which were then not genotyped due to recent fever / allergy. ${ }^{\circ}$ denotes significance in comparing PT to NPT; * denotes significance in comparing $\mathrm{T} 1$ to $\mathrm{T} 0$. 
NPT: genotipe DAT 10/10

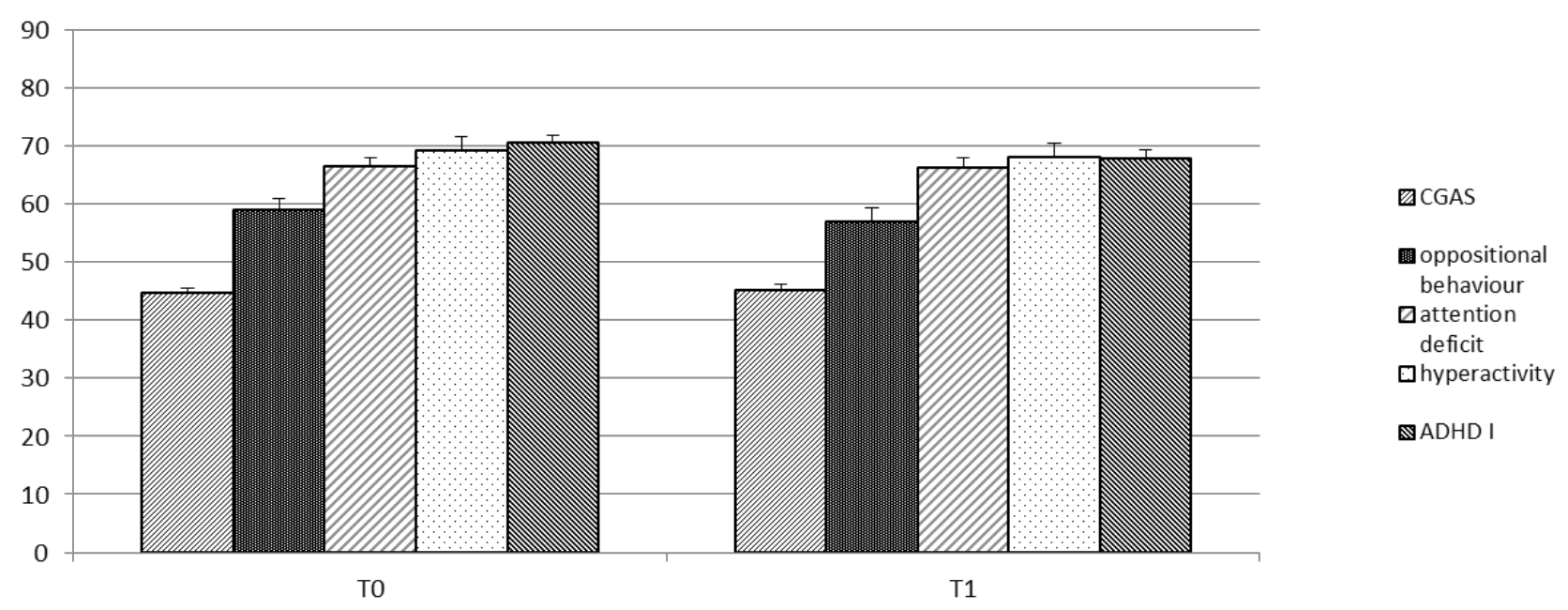

NPT: genotipe DAT 9/x

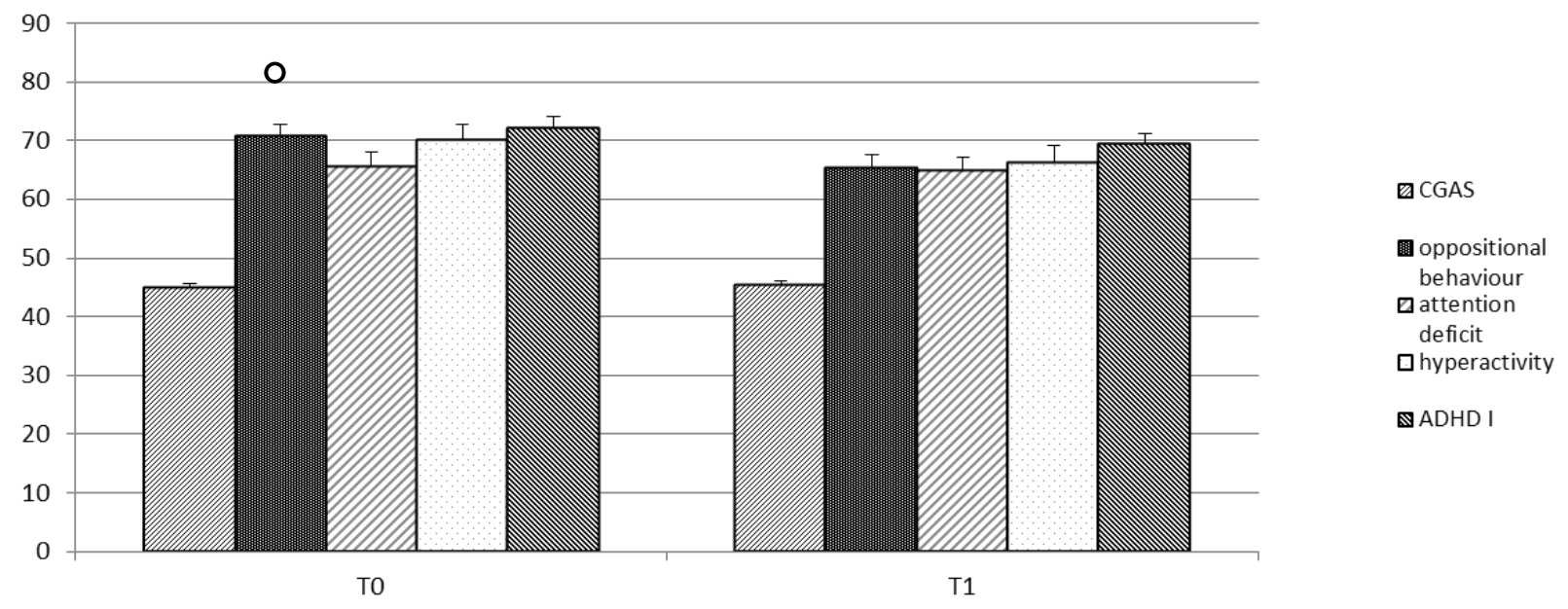

Figure 2

(upper panel) - Psychometric scales in genotype 10/10 children in NPT group ( $\mathrm{n}=12$ ).

(lower panel) - Psychometric scales in genotype 9/x children of the NPT group ( $\mathrm{n}=11$ ). 


\section{PT: genotipe DAT 10/10}

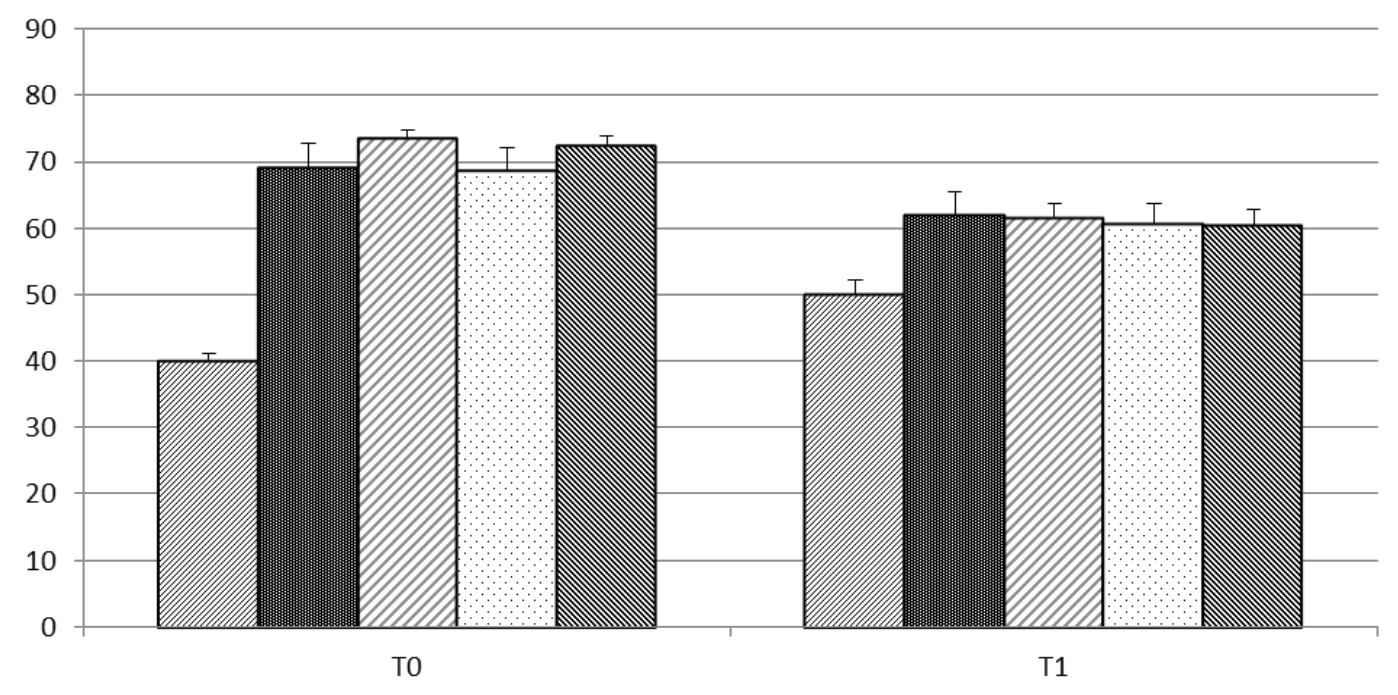

चCGAS

国 oppositional

behaviour

$\square$ attention

deficit

] hyperactivity

$\mathbb{A D H D} \mathbf{I}$

\section{PT: genotipe DAT 9/x}

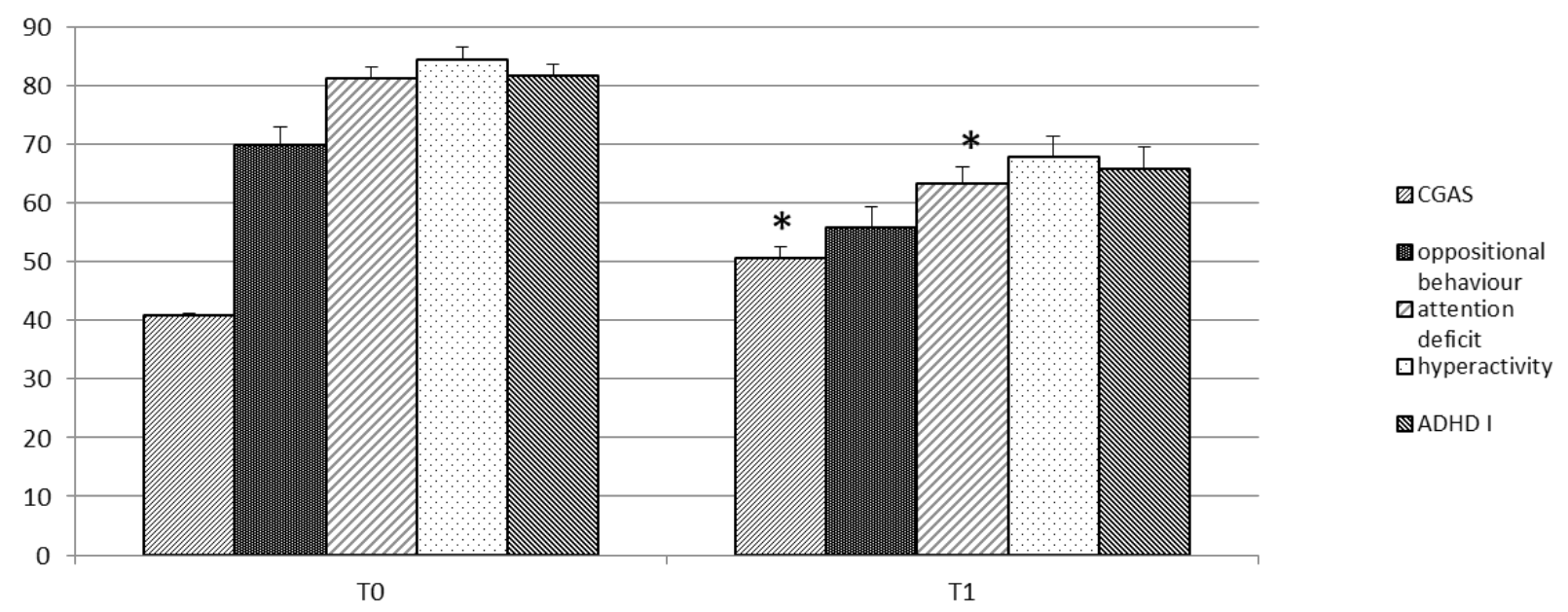

Figure 3

(upper panel) - Psychometric scales in genotype 10/10 children in PT group ( $\mathrm{n}=3$ ).

(lower panel) - Psychometric scales in genotype 9/x children of the PT group ( $\mathrm{n}=4$ ). 

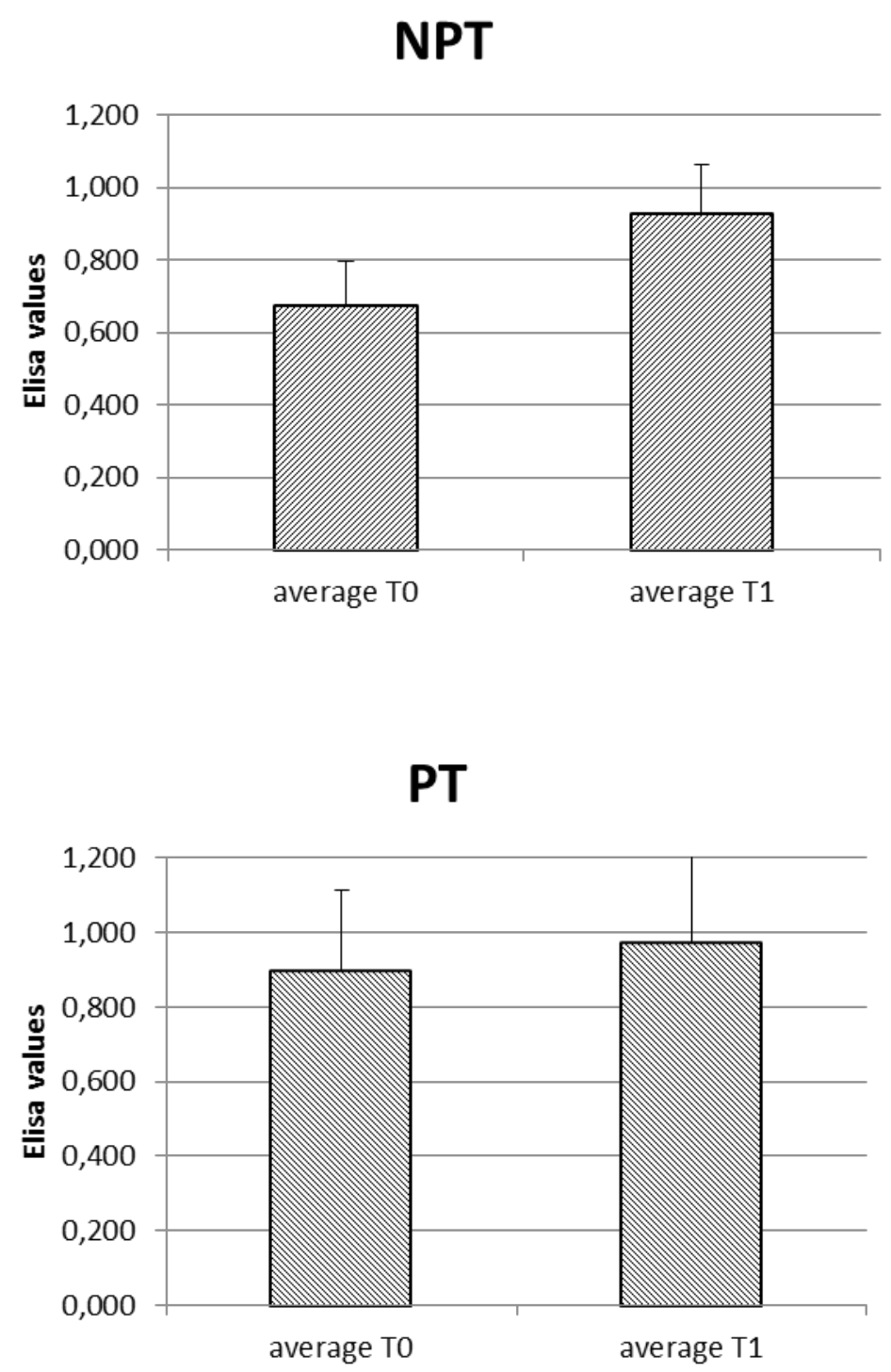

Figure 4

(upper panel) - hDAT aAbs titer in recruited ADHD children not assigned in a pharmaco-therapy (NPT, N=32). (lower panel) - hDAT aAbs titer in recruited ADHD children assigned to pharmacological treatment (PT, N=14). Data include also ADHD children which were then not genotyped due to recent fever / allergy. 
NPT: genotipe DAT

\section{$10 / 10$}

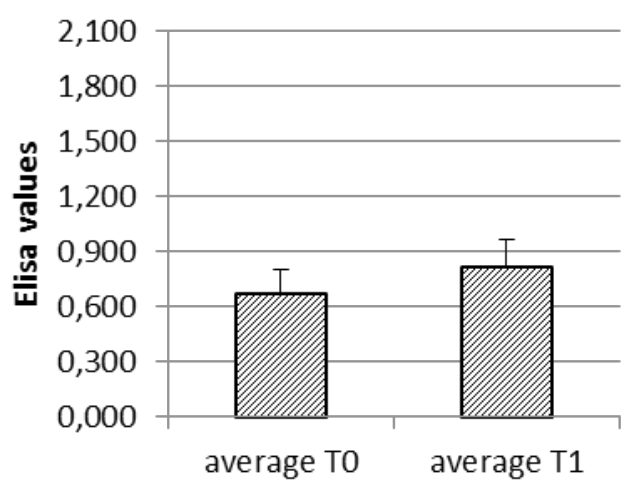

Fig. 5a

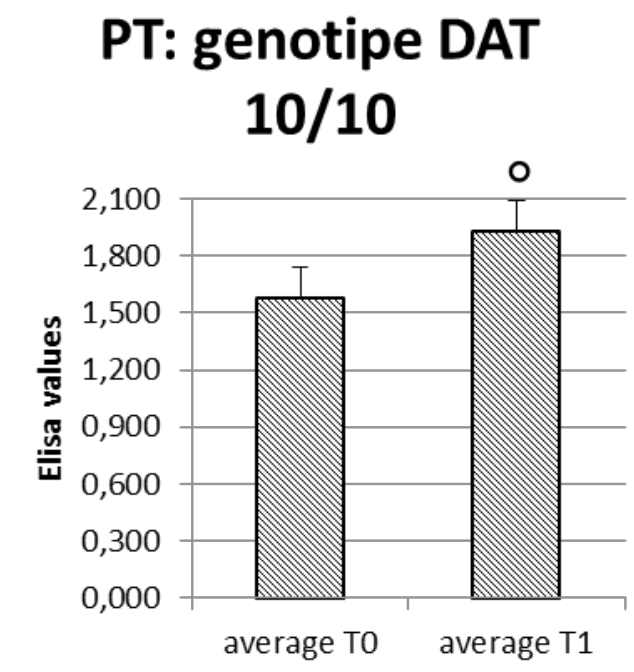

Fig. 5c
NPT: genotipe DAT $9 / x$

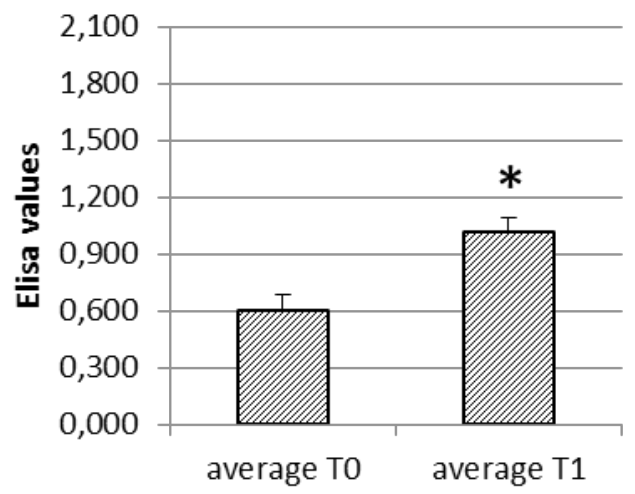

Fig. $5 b$

\section{PT: genotipe DAT $9 / x$}

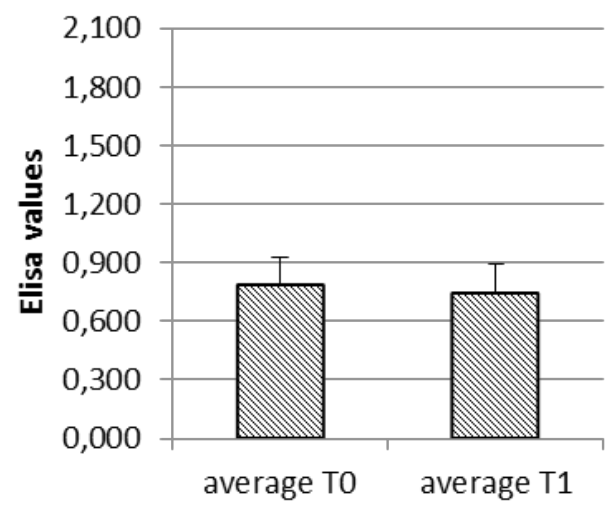

Fig. $5 d$

Figure 5

(panel a) - hDAT aAbs in children of NPT group, genotype DAT 10/10 (n=12). (panel b) hDAT aAbs in children of NPT group, genotype DAT 9/x ( $\mathrm{n}=11)$. (panel $\mathrm{c})-\mathrm{hDAT}$ aAbs in children in the PT group, genotype DAT 10/10 ( $n=3)$. (panel d) - hDAT aAbs in children in the PT group, genotype DAT 9/x $(n=4) .{ }^{\circ}$ denotes significance in comparing PT to NPT; * denotes significance in comparing $\mathrm{T} 1$ to $\mathrm{T} 0$. 